\title{
Nerve Growth Factor Pathobiology During the Progression of Alzheimer's Disease
}

\begin{abstract}
Elliott J. Mufson ${ }^{1 *}$, Scott E. Counts ${ }^{2}$, Stephen D. Ginsberg ${ }^{3,4}$, Laura Mahady ${ }^{1}$, Sylvia E. Perez ${ }^{1}$, Stephen M. Massa ${ }^{5}$, Frank M. Longo ${ }^{6}$ and Milos D. Ikonomovic ${ }^{7}$

${ }^{1}$ Department of Neurobiology and Neurology, Department of Neurobiology, and Department of Neurological Sciences, Alzheimer's Disease Laboratory, Barrow Neurological Institute, St. Joseph's Medical Center, Phoenix, AZ, United States, 2 Translational Science and Molecular Medicine Michigan State University College of Human Medicine, Grand Rapids, MI, United States, ${ }^{3}$ Center for Dementia Research, Nathan Kline Institute, Orangeburg, NY, United States, ${ }^{4}$ Department of Psychiatry, Department of Neuroscience, and Physiology and NYU Neuroscience Institute, New York University Langone Medical Center, New York, NY, United States, ${ }^{5}$ Department of Neurology, San Francisco VA Health Care System, University of California, San Francisco, San Francisco, CA, United States, ${ }^{6}$ Department of Neurology and Neurological Sciences, Stanford University School of Medicine, Stanford, CA, United States, ${ }^{7}$ Department of Neurology and Department of Psychiatry, Geriatric Research Education and Clinical Center, VA Pittsburgh Healthcare System, University of Pittsburgh, Pittsburgh, PA, United States
\end{abstract}

The current review summarizes the pathobiology of nerve growth factor (NGF) and its cognate receptors during the progression of Alzheimer's disease (AD). Both transcript and protein data indicate that cholinotrophic neuronal dysfunction is related to an imbalance between TrkA-mediated survival signaling and the NGF precursor (proNGF)/p75 ${ }^{\text {NTR }}$-mediated pro-apoptotic signaling, which may be related to alteration in the metabolism of NGF. Data indicate a spatiotemporal pattern of degeneration related to the evolution of tau pathology within cholinotrophic neuronal subgroups located within the nucleus basalis of Meynert (nbM). Despite these degenerative events the cholinotrophic system is capable of cellular resilience and/or plasticity during the prodromal and later stages of the disease. In addition to neurotrophin dysfunction, studies indicate alterations in epigenetically regulated proteins occur within cholinotrophic nbM neurons during the progression of $A D$, suggesting a mechanism that may underlie changes in transcript expression. Findings that increased cerebrospinal fluid levels of proNGF mark the onset of $\mathrm{MCl}$ and the transition to $\mathrm{AD}$ suggests that this proneurotrophin is a potential disease biomarker. Novel therapeutics to treat NGF dysfunction include NGF gene therapy and the development of small molecule agonists for the cognate prosurvival NGF receptor TrkA and antagonists against the pan-neurotrophin p75 NTR death receptor for the treatment of AD.

Keywords: Alzheimer, nerve growth factor, mild cognitive impairment, epigenetics, neurotrophin receptors, biomarker

\section{INTRODUCTION}

Alzheimer's disease (AD) is a progressive and fatal age-associated brain disorder characterized clinically by memory decline, impairment of activities of daily living, neuropsychiatric symptoms, and other behavioral disturbance. Prevalence reports indicate that approximately 18 million people have AD worldwide, with >5.8 million people in the United States (Alzheimer's Association, 2019). 
The percentage of cases increases twofold with approximately every 5 years of an increase in age, indicating that $1 \%$ of individuals 60 years of age and approximately $30 \%$ of people 85 years of age will exhibit the disease. Lacking significant intervention, the number of symptomatic people in the United States will increase to 13.8 million by midcentury (2019). The cost of caring for those with AD will exceed 100 billion United States dollars yearly (Christensen, 2007; Wimo, 2007; Wimo et al., 2017). These alarming statistics stress the overwhelming importance of developing effective treatments for use in the early or prodromal stages of AD.

\section{PRODROMAL AD}

Alzheimer's disease has an extensive preclinical stage, possibly as early as 15-20 years before the onset of clinical symptoms (Sperling et al., 2014) (Figure 1). Mild cognitive impairment (MCI), a term now synonymous with prodromal $\mathrm{AD}$, is an intermediate phase between normal brain aging and frank dementia when neurofibrillary tangles (NFTs) and amyloid-beta peptide $(\mathrm{A} \beta)$ lesions are increased in comparison to those with no cognitive impairment (NCI) (Guillozet et al., 2003; Markesbery et al., 2006; Markesbery, 2010). MCI as a clinical concept was developed from memory clinics, which evaluated milder demented subjects from longitudinal investigations of older cohorts who were tested annually for cognitive status. Such investigations demonstrated that many with earlier, milder cognitive decline failed to show impairment in two cognitive domains as required for an NINDS/ADRDA AD diagnosis (McKhann et al., 1984). These people were defined with an amnestic disorder and termed amnestic MCI (aMCI) (Petersen et al., 1999). Although memory clinics suggested that aMCI was the more common type of MCI leading to $\mathrm{AD}$, it was evident that this entity comprised a minor but a significant aspect of this clinical classification. Overall, the clinical diagnosis of MCI encompasses a heterogeneous population of patients that includes those with isolated memory problems, classified as single domain aMCI, while those with a memory deficit and other cognitive domain impairments are categorized as multi-domain MCI (mdMCI) (Petersen, 2004; Johnson et al., 2010). Amnestic MCI cases are at a greater risk of developing AD (Petersen, 2004; Johnson et al., 2010). A significant proportion of elderly people clinically diagnosed with NCI or with MCI display amyloid plaque and NFT pathology similar to that seen in AD, challenging the pathologically-based concept that these lesions alone hasten dementia onset (Mufson and Kordower, 1999; Price and Morris, 1999; Markesbery, 2010; Mufson et al., 2016a,b).

\section{CLASSIC AD LESIONS}

By the twentieth century, neuropathological investigations reported the existence of abnormal extracellular plaques in brains obtained postmortem from older adults with dementia (Blocq and Marinesco, 1892). The German psychiatrist, Dr. Alois Alzheimer, treated Auguste Deter, a 51-year old woman, who presented with signs of paranoia and memory impairment and died 5 years after diagnosis. At autopsy, her brain appeared atrophic, with a loss of neurons, and contained NFTs and senile plaques (SPs). Dr. Emil Kraepelin termed this triad of features "Alzheimer's disease." SPs found in the extracellular matrix consist of insoluble fibrils of $A \beta$, produced from a larger transmembrane amyloid- $\beta$ precursor protein (APP) by the successive cleavage by the $\beta$-site APP cleaving enzyme 1 (BACE1) and the intramembrane $\gamma$-secretase complex (Shoji et al., 1992; Thinakaran and Koo, 2008). NFTs consist of intracellular aggregates of hyperphosphorylated tau protein (Trojanowski et al., 1993; Yoshiyama et al., 2013). Although SPs and NFTs are considered the defining pathological hallmarks of $\mathrm{AD}$, Dr. Alzheimer wrote that “...the plaques are not the cause of senile dementia, but only an accompanying feature of senile involution of the central nervous system" (Alzheimer, 1911). Despite this statement, the AD research field has been driven by the "amyloid cascade hypothesis" (Hardy and Selkoe, 2002) and treatment strategies continue to revolve around the development of anti-amyloid drugs to remove plaque deposition. However, virtually all anti-amyloid clinical trials have not met their primary end-point, the improvement of cognition (Hampel et al., 2015, 2018). This lack of drug efficacy lends support to the concept that amyloid may be an early biomarker of AD but not necessary for a clinical decline. More likely AD is a multifaceted polygenic disease of which amyloid is a partner in the pathogenesis of this disease. Contrary to the amyloid hypothesis, a large body of literature suggests the loss of cognition involves the selective vulnerability of multiple neurotransmitter pathways leading to a massive cortical disconnection syndrome.

\section{CHOLINOTROPHIC BASAL FOREBRAIN DEFECTS DURING THE PROGRESSION OF AD}

For over 30 years degeneration of cholinergic basal forebrain (CBF) neurons, which innervate the entire neocortex and hippocampus (Figures 2A-D) has been investigated as a key neurotransmitter system affected early in the disease that may be a target for AD treatment (Hampel et al., 2018). The "cholinergic hypothesis" of AD (Bartus et al., 1982) gained momentum with the finding that acetylcholinesterase inhibitors (AChEIs) have significant symptomatic effects in AD patients (Summers et al., 1986) This led to the development of a larger family of acetylcholinesterase inhibitors (AChEIs) (Hampel et al., 2018) (Figures 2E-G), which remains one of the few classes of FDA approved drugs for the treatment of AD (Johannsen, 2006; Mangialasche et al., 2010; Hampel et al., 2018). For example, the AChEI, donepezil, has been shown to reduce basal forebrain atrophy within the nucleus basalis of Meynert $(\mathrm{nbM})$ and the medial septum/diagonal band in prodromal $\mathrm{AD}$, demonstrating a structural effect (Cavedo et al., 2017) as well as symptomatic relief.

Recently, there has been a resurgence of interest in the $\mathrm{CBF}$ projection system in the field of early-onset dementia (Douchamps and Mathis, 2017; Hampel et al., 2018). Imaging 


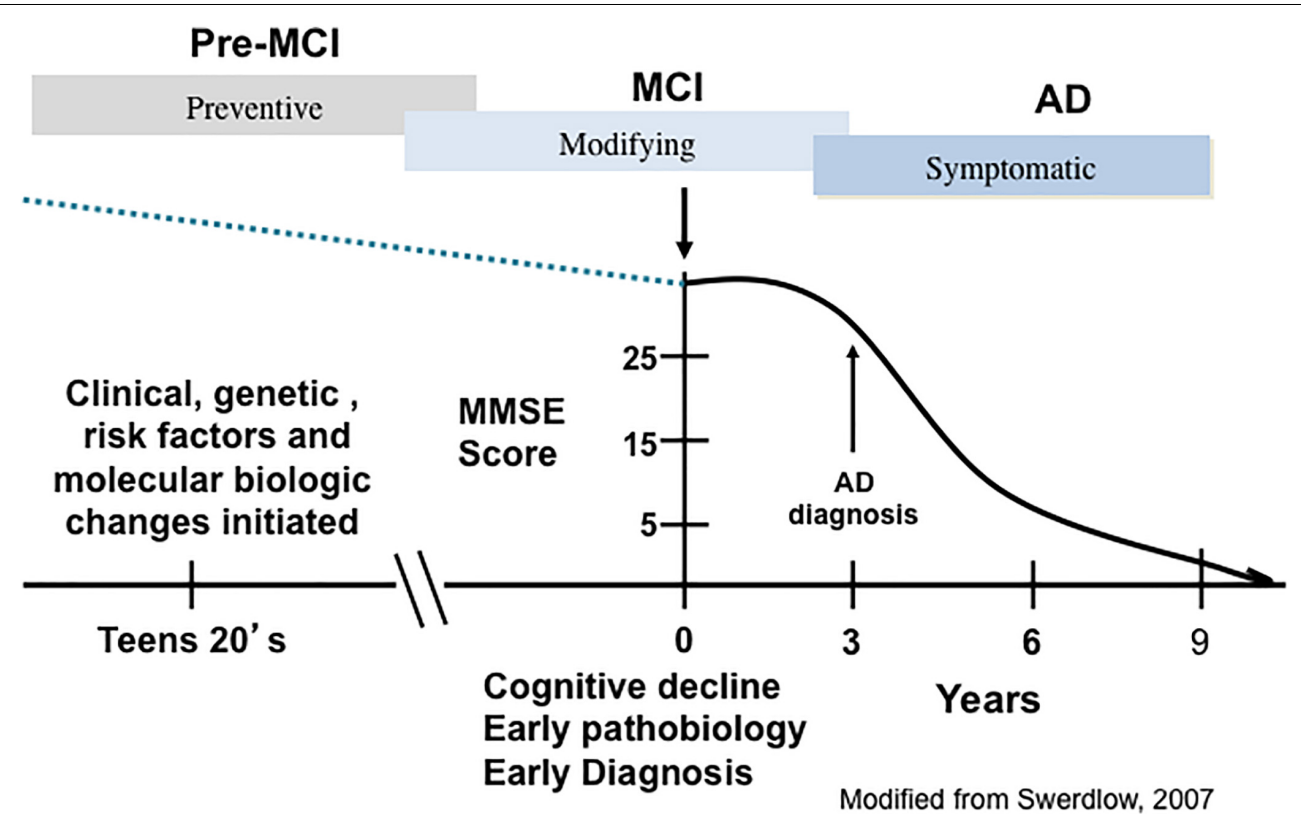

FIGURE 1 | A schematic illustration depicting the trajectory of the clinical and pathological progression of Alzheimer's disease (AD). PreMCI, pre-mild cognitive impairment; $\mathrm{MCl}$, mild cognitive impairment.

studies provide evidence of the importance of dysregulated basal forebrain circuitry in signaling related to cognitive decline (Ballinger et al., 2016), dysregulation of the default mode network (DMN) critical for executive function, episodic memory (Nair et al., 2018), and propagation of cortical atrophy early in the evolution of the disease (Schmitz and Nathan Spreng, 2016), and as a pre-symptomatic biomarker for AD (Ho et al., 2008; Grothe et al., 2012). This renewed interest in cholinergic cortical projection neurons to the DMN and other cortical sites during the onset of $\mathrm{AD}$ underscores the critical need to understand the mechanistic factors underlying dysfunction of this projection system.

\section{NERVE GROWTH FACTOR DURING THE PROGRESSION OF AD}

Since Ramon y Cajal suggested that brain cells require "special food," researchers have searched for growth-stimulating agents that play a role in neuronal survival (Henry, 1998). Levi-Montalcini and Cohen (Levi-Montalcini, 2000), received the Nobel Prize for their discoveries showing that the trophic substance nerve growth factor (NGF) underlies the selective survival of cultured neurons. They were the first to suggest the neurotrophic hypothesis of neuronal survival. NGF is a product of a single gene found on chromosome 1, which gives rise to a 27 kiloDalton $(\mathrm{kDa})$ and a $35 \mathrm{kDa}$ proNGF precursor protein (Francke et al., 1983; Edwards et al., 1988), which are proteolytically cleaved to a mature biologically active peptide (Edwards et al., 1988; Lee et al., 2001). ProNGF, not mature NGF, is the primary form found in the human brain (Fahnestock et al., 2001). NGF binds to its cognate tropomyosin-related kinase A (TrkA) receptor and the p75 pan-neurotrophin receptor (p75 ${ }^{\text {NTR }}$ ) (Ibanez, 2002; Chao, 2003; Kaplan and Miller, 2004). NGF binding to TrkA activates downstream survival pathways by activating Akt (Ulrich et al., 1998) while proNGF and $\mathrm{p} 75^{\mathrm{NTR}}$, together with its co-receptors sortilin (Nykjaer et al., 2004) and neurotrophin receptor homolog-2 (NRH2) (Murray et al., 2004) activate the c-Jun N-terminal protein kinase (JNK) related to cellular apoptosis (Nykjaer et al., 2005) (Figure 3). Clinical trials have shown that NGF has therapeutic potential to enhance CBF survival and neuroplasticity in AD (Tuszynski et al., 1990; Tuszynski and Blesch, 2004; Tuszynski et al., 2015).

\section{NGF AND THE PROGRESSION OF AD}

Although for many years it was hypothesized that cholinotrophic basal forebrain cortical and hippocampal projection neurons degenerate due to loss of NGF in AD (Hefti and Mash, 1989; Tuszynski et al., 1990; Smith et al., 1999), studies reported unchanged (Goedert et al., 1989; Allen et al., 1991; Murase et al., 1993; Jette et al., 1994), decreased (Hellweg et al., 1998) or increased (Crutcher et al., 1993; Scott et al., 1995; Fahnestock et al., 1996; Narisawa-Saito et al., 1996; Hellweg et al., 1998; Hock et al., 2000) NGF levels using tissue from severe AD subjects. However, NGF levels were preserved in five cortical regions (superior frontal, superior temporal, middle temporal, anterior cingulate, and inferior parietal cortex) and hippocampus in people who came to autopsy with a clinical diagnosis of $\mathrm{MCI}$, mild AD, and severe AD (Figure 4) (Mufson et al., 2003). In contrast, others report an increase in cortical and hippocampal $\mathrm{Ngf}$ mRNA and protein in end-stage $\mathrm{AD}$, where volume loss 

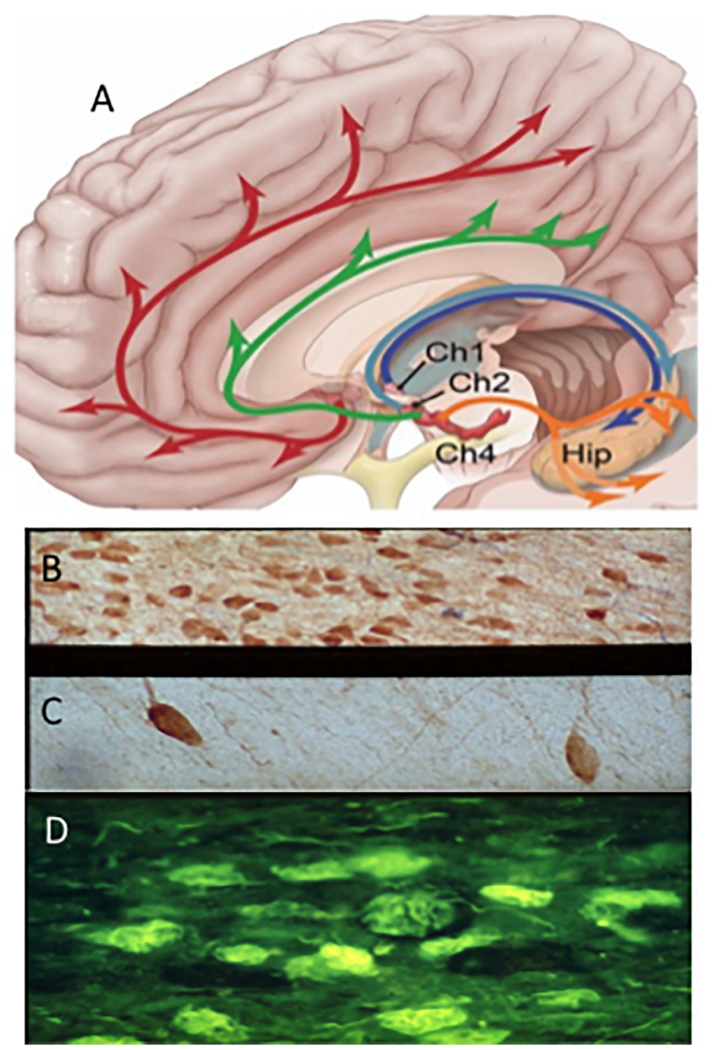
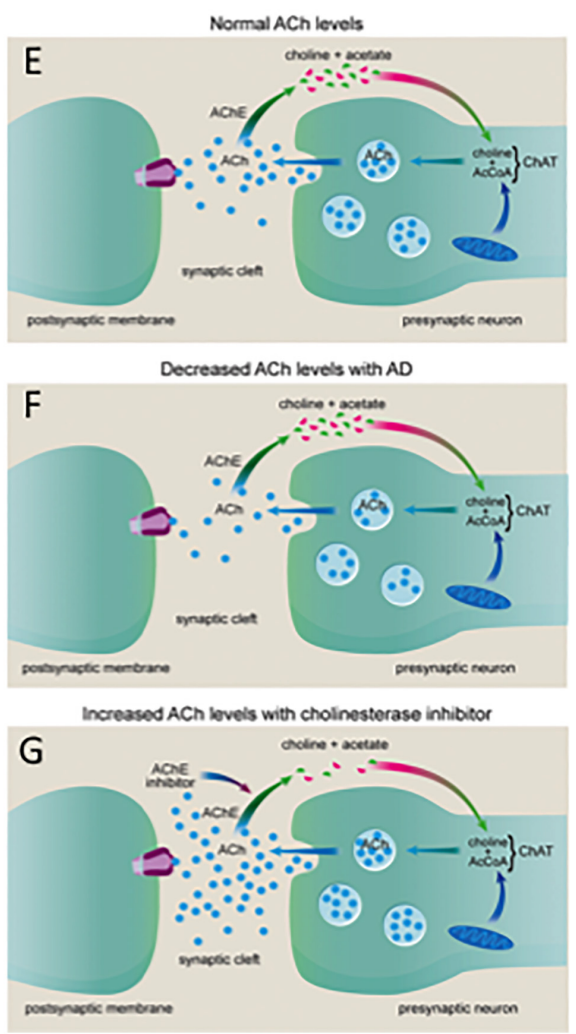

FIGURE 2 | (A) Schematic drawing of the cholinotrophic cortical and hippocampal projection systems. Photomicrographs showing images of cholinotrophic neurons in an aged control (B), reduction in AD (C) and thioflavin (yellow) tangle-bearing p75 NTR neurons (dark blue) (D) in AD. Cartoon showing changes in acetylcholine (ACh) between a normal $(\mathbf{E})$ and $A D(\mathbf{F})$ and the effect of acetylcholinesterase inhibitors at the cholinergic synapse (G). Ch1, medial septal cholinergic cell group; Ch2, vertical limb of the diagonal band cholinergic cell group Ch4; nucleus basalis cholinergic cell group and Hip, hippocampus. Red and green arrows indicate bilateral Ch4 projections; dark blue and light blue arrows indicate septal/diagonal band projections to the hippocampus.

could lead to increased concentrations of NGF per weight or volume (Crutcher et al., 1993; Jette et al., 1994; Scott et al., 1995; Fahnestock et al., 1996; Narisawa-Saito et al., 1996; Hellweg et al., 1998; Hock et al., 2000) or the translation from $\mathrm{Ngf}$ to encoded NGF protein may be compromised or expression levels differ between $\mathrm{AD}$ cases. We reported a wide range of NGF activity in a cohort ranging from early to late-onset $\mathrm{AD}$ cases and some of the highest and lowest levels of NGF were seen in end-stage AD cases (Scott et al., 1995), suggesting that within a given cohort, NGF levels can be differentially affected by age at disease onset or differences in disease process. In this study, there was no relationship between cortical choline acetyltransferase (ChAT) activity, the rate-limiting enzyme for acetylcholine synthesis, and levels of NGF, nor between reduced numbers of ChAT- (Gilmor et al., 1999), TrkA- (Mufson et al.,

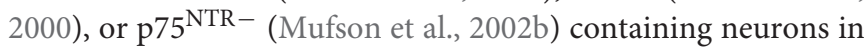
MCI and mild AD. Moreover, the lack of a correlation between the apolipoprotein $\varepsilon 4$ genotype and NGF levels is interesting, since ApoE $\varepsilon 3$ and $\varepsilon 4$ alleles are reported to be associated with a greater decrease in cholinergic markers in end-stage AD (Poirier et al., 1995). Although over $90 \%$ of the severe AD cases we examined from the Rush Religious Orders Study (RROS) contained at least one ApoE $\varepsilon 4$ allele, NGF levels did not differ across the clinical groups evaluated (Mufson et al., 2003). These observations suggest that ApoE $\varepsilon 4$ genotype does not directly affect the metabolism of NGF.

\section{EXPRESSION OF NGF RECEPTORS DURING THE PROGRESSION OF AD}

Cholinergic basal forebrain neuron function is dependent upon the binding of NGF to its cognate receptor TrkA, as well as its pan-neurotrophin $\mathrm{p} 75^{\mathrm{NTR}}$, which lends support to the suggestion that dysregulation of NGF and its receptors underlie cholinergic neuron dysfunction in $\mathrm{AD}$. TrkA receptors and p $75^{\text {NTR }}$ are produced within the perikarya of CBF neurons and anterogradely transported to the cortex and hippocampus the sties of NGF production (Schwab et al., 1979). Within CBF neurons, mature NGF binds to the TrkA receptor, activating signal transduction pathways that regulate neuronal survival induced by NGF (Kaplan and Miller, 2004). However, $\mathrm{p} 75^{\mathrm{NTR}}$ is a positive modulator of NGF/TrkA binding (Kaplan and Miller, 2004), and exhibits several context-dependent functions including the stimulation of apoptotic or cell death pathways (Bamji et al., 1998; Yoon et al., 1998; Frade, 2000; 


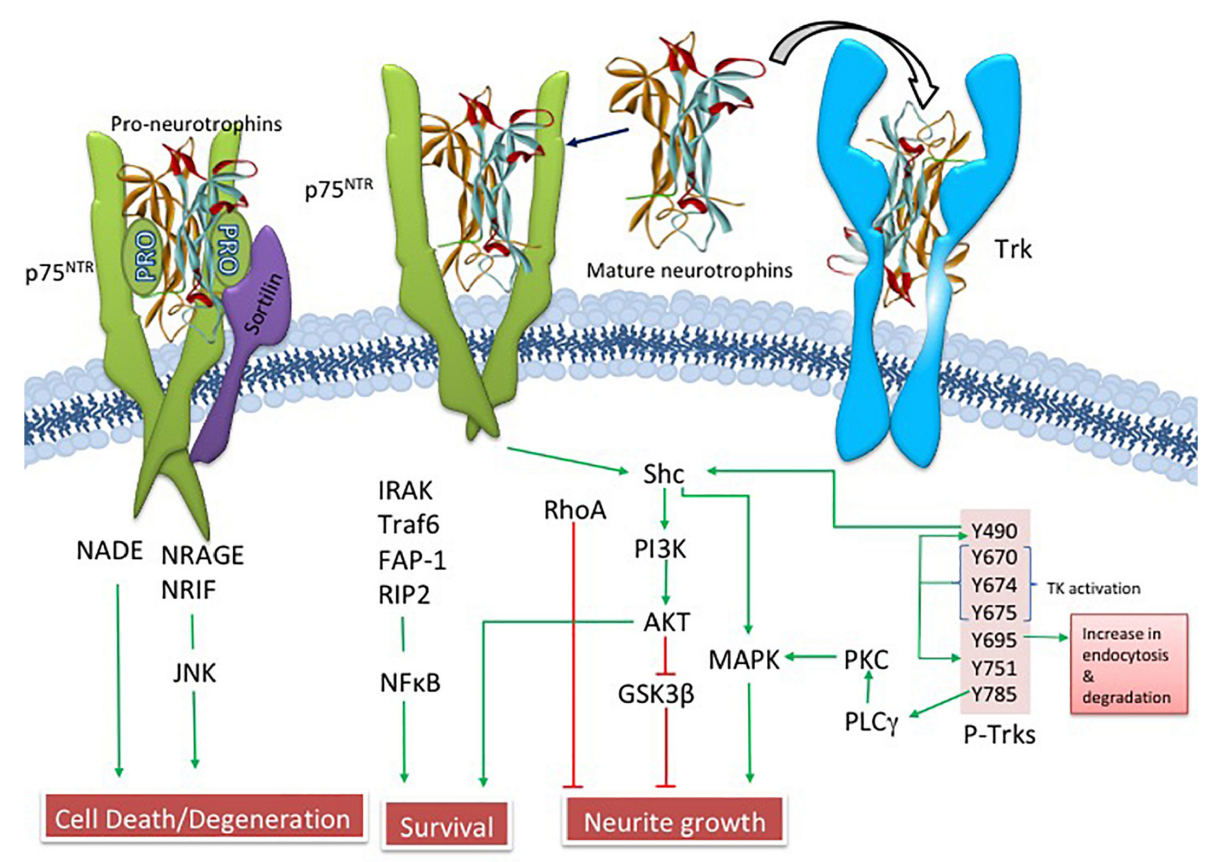

FIGURE 3 | Illustration showing NGF related upstream and downstream pathways.
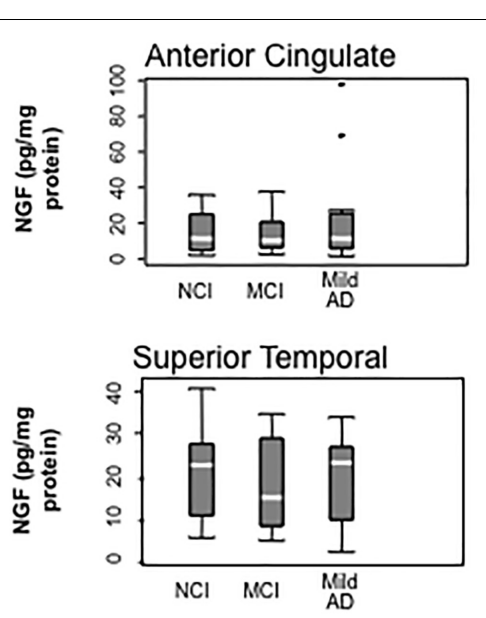
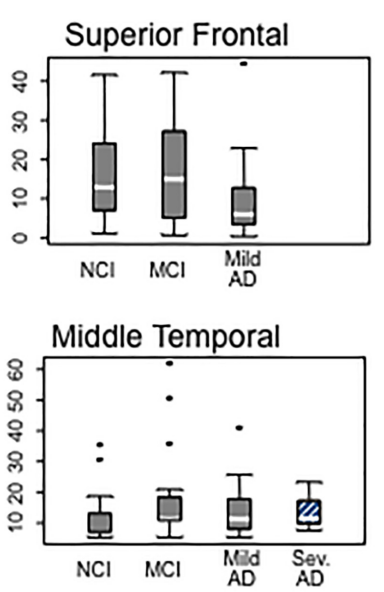
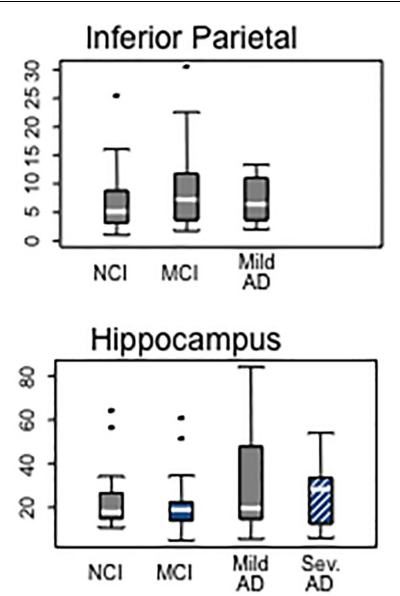

FIGURE 4 | Box plots showing the stability of NGF protein levels with the cortex and hippocampus during the progression of AD. Reproduced from Mufson et al. (2003).

Friedman, 2000; Lee et al., 2001; Roux and Barker, 2002). In this regard, the specific downstream effects of $\mathrm{p} 75^{\mathrm{NTR}}$ are dependent upon its interaction with various receptor chaperones (Mamidipudi and Wooten, 2002; Nykjaer et al., 2004; Teng and Hempstead, 2004).

In order to evaluate whether the number of $\mathrm{CBF}$ neurons containing NGF receptors is altered early in the progression of $\mathrm{AD}$, we examined tissue from RROS subjects clinically categorized as NCI, MCI, or AD (Gilmor et al., 1999). Interestingly, the numbers of ChAT-containing neurons were stable in MCI and mild AD, while TrkA- and p $75^{\mathrm{NTR}}$-immunoreactive neurons were significantly decreased compared to NCI, indicating a phenotypic downregulation of receptors supporting $\mathrm{CBF}$ function rather than frank neuronal degeneration in MCI (Gilmor et al., 1999) (Figure 5). The phenotypic loss of cholinotrophic markers due to atrophy, rather than overt cholinergic cell loss, is consistent with animal model studies of septal cholinergic neuron axotomy via fimbria-fornix transection and excitotoxicity (Hefti, 1986; Williams et al., 1986; Ginsberg and Martin, 1998). In AD, reduced cortical TrkA levels positively correlated with lower cognitive performance as assessed by the Mini-Mental State 


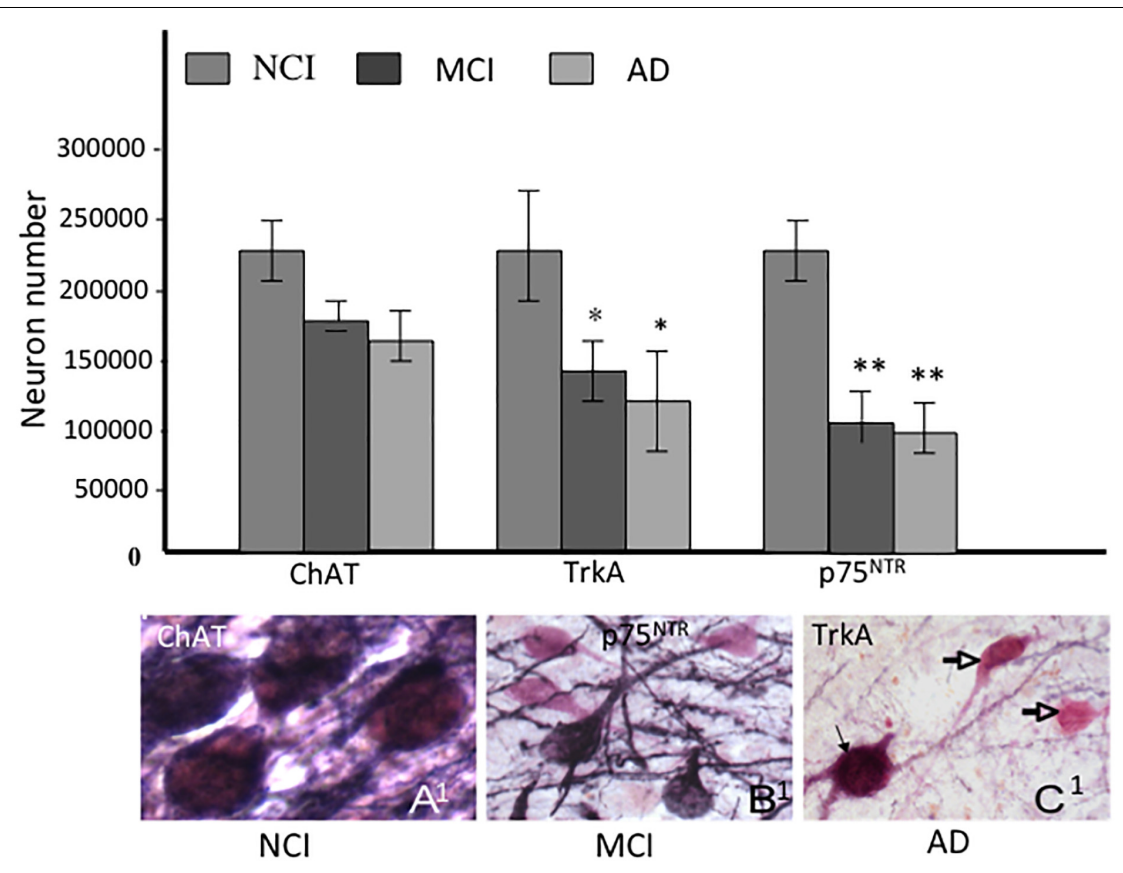

FIGURE 5 | Histograms showing the differential reduction in cholinergic, TrkA and p75 ${ }^{\mathrm{NTR}}$ immunoreactive neurons during the progression of $A D$. $\mathrm{A}^{1}$ - $\mathrm{C}^{1}$. Photomicrographs of dual labeled nucleus basalis neurons showing phenotypic downregulation of p75 NTR (dark blue) compared to ChAT (pink) positive neurons between no cognitive impairment (NCl), mild cognitive impairment (MCl) and AD. Note the loss of p75 NTR immunoreactive staining of ChAT-positive cells in MCl and $\mathrm{AD}$ (open arrows). Black arrow indicates dual stained neuron in $\mathrm{AD}\left(\mathrm{C}^{1}\right){ }^{*}$ and $* *$ indicate $p<0.05$ and 0.01 , respectively.

Exam (MMSE) (Counts et al., 2004), suggesting that decreased nbM and cortical NGF receptor protein levels may mark the early onset of AD.

\section{CORTICAL PRONGF LEVELS DURING THE PROGRESSION OF AD}

Alterations in proNGF, the NGF precursor protein, has received extensive study in clinical pathological investigation of components of the cortical DMN, which includes frontal cortex, posterior cingulate, precuneus, superior temporal cortex (Perez et al., 2015), and the hippocampus (Mufson et al., 2012b), which contribute to cognitive dysfunction during the progression of AD (Sperling et al., 2014). ProNGF isolated from AD cortex induces apoptosis in neuronal cell cultures by interacting with p $75^{\mathrm{NTR}}$ via a mechanism dependent upon $\gamma$-secretase shedding of the receptor, whereas proNGF isolated from control brain does not activate apoptosis (Pedraza et al., 2005). ProNGF levels are increased in the lateral parietal cortex of patients who died with a clinical diagnosis of MCI or mild AD compared to those with NCI (Peng et al., 2004). In contrast, precuneus proNGF levels were stable until end-stage AD (Perez et al., 2015), similar to that of the frontal cortex (Fahnestock et al., 2001, 2004; Podlesniy et al., 2006) and hippocampus (Al-Shawi et al., 2008; Mufson et al., 2012b), all of which suggest alterations of proNGF in the diseased brain. Western blotting found no changes in the levels of TrkA, p75 ${ }^{\mathrm{NTR}}$ and the co-receptor, sortilin within the precuneus (Perez et al., 2015), and hippocampus
(Mufson et al., 2010) across clinical groups. ProNGF binds with a higher affinity to $\mathrm{p} 75^{\mathrm{NTR}}$, which is enhanced in the presence of sortilin to induce apoptosis (Lee et al., 2001; Nykjaer et al., 2004; Pedraza et al., 2005; Al-Shawi et al., 2008). Homeostatic regulation of NGF receptors, combined with the binding of proNGF to TrkA (albeit with less affinity than mature NGF), results in the activation of downstream pathways involved in CBF neuron function (Fahnestock et al., 2001, 2004) as well as the induction of neurotrophic activity via the binding with less affinity to the TrkA receptor (Fahnestock et al., 2001, 2004). The finding that $\mathrm{p} 75^{\mathrm{NTR}}$ levels remain stable in the precuneus and other cortical regions (Counts et al., 2004; Mufson et al., 2012b) during the onset of $\mathrm{AD}$ may be related to the demonstration of a de novo appearance of $\mathrm{p} 75^{\mathrm{NTR}}$ cortical neurons in $\mathrm{AD}$ (Mufson and Kordower, 1992).

The pro-apoptotic effect(s) of $\mathrm{p} 75^{\mathrm{NTR}}$-mediated proNGF signaling is dependent on interactions with $\mathrm{p} 75^{\mathrm{NTR}}$ and sortilin, a Vps10p domain trafficking protein that acts as a cell surface co-receptor with $\mathrm{p} 75^{\mathrm{NTR}}$ to mediate proNGF-activated cell death. This family of receptors is gaining importance, due to its potential involvement in AD (Nyborg et al., 2006). Sortilin activates $\mathrm{p} 75^{\mathrm{NTR}}$-induced apoptosis following proNGF treatment (Nykjaer et al., 2004), suggesting a role in cell death (Mamidipudi and Wooten, 2002; Roux and Barker, 2002). Blocking this binding event precludes binding of proNGF to $\mathrm{p} 75^{\mathrm{NTR}}$ and subsequent cell degeneration (Bronfman and Fainzilber, 2004; Kaplan and Miller, 2004; Nykjaer et al., 2004; Teng et al., 2005). It is possible that $\mathrm{p} 75^{\mathrm{NTR}}$ signaling in response to proneurotrophins depends upon the identity and 
efficacy of the bound co-receptor. Notably, cortical levels of sortilin remain stable, similar to $\mathrm{p} 75^{\mathrm{NTR}}$ during the progression of AD. Perhaps pro-survival or pro-apoptotic signaling in $\mathrm{CBF}$ neurons is dependent upon changes in the stoichiometry of TrkA, $\mathrm{p} 75^{\mathrm{NTR}}$, the availability of select co-receptors, and the physiological role of proNGF within different milieus during the early stage of AD. Shifting the balance of these factors may change the response that proNGF binding activates within $\mathrm{CBF}$ neurons during the progression of $\mathrm{AD}$ (Figure 6). Defining these interactions will be key to the development of neurotrophic strategies for dementia (Bruno et al., 2004; Longo et al., 2007). If proNGF binds $\mathrm{p} 75^{\mathrm{NTR}}$ in vivo and induces apoptosis (Lee et al., 2001; Nykjaer et al., 2004), it will be crucial to develop drugs that block proNGF binding to $\mathrm{p} 75^{\mathrm{NTR}}$. In contrast, if proNGF binds with TrkA to induce cell survival (Fahnestock et al., 2004), then the development of drugs that enhance this interaction could provide neuroprotection in $\mathrm{AD}$.

\section{NGF METABOLIC PATHWAYS DURING THE PROGRESSION OF AD}

Defects in the metabolic pathways regulating the maturation and degradation of the NGF/proNGF complex may play a key role in CBF dysfunction. Recently, a protease cascade, which converts proNGF to mature mNGF and degrades mNGF in the extracellular space by the coordinated activity of plasminogen, tissue plasminogen activator (tPA), neuroserpin, matrix metalloproteinase 9 (MMP-9) and tissue inhibitor of matrix metalloproteinase 1 (TIMP-1) was shown to be defective in $\mathrm{AD}$ (Bruno and Cuello, 2006). In this regard, the upregulation of MMP-9 protein levels and activity were reported in the frontal and parietal cortex in MCI and $\mathrm{AD}$, which was inversely associated with cognitive performance (Bruno et al., 2007), and may drive changes in NGF/proNGF activity (Figure 7). We suggest that increased proMMP-9 and MMP-9 compromises NGF support of CBF neurons during the transition from NCI to MCI (Figure 7). Interestingly, a similar increase in cortical proNGF (Iulita et al., 2014) and reduction in TrkA-positive CBF neurons (Sendera et al., 2000) has been reported in Down syndrome (DS), suggesting an overlap in NGF neurotrophic dysregulation in these disorders. Both AD and DS cases display cortical SP and NFT pathology and develop dementia by midlife (Mann and Esiri, 1989), further connecting these neurological conditions. It has been suggested that levels of metalloproteinases (MMPs) in blood, urine, and cerebrospinal fluid (CSF) may act as potential biomarkers for AD (Zucker et al., 1999; Lorenzl et al., 2003, 2008). It is of interest to examine whether or not MMPs are dysregulated in the precuneus, allowing for the stable metabolic $\mathrm{NGF}$ /proNGF complex regulation early in AD. Notably, proNGF levels were not associated with increased soluble $A \beta_{1-42}$ or fibrillar $\mathrm{A} \beta[3 \mathrm{H}]$ Pittsburgh Compound $\mathrm{B}$ (PiB) binding, but instead with compact/cored 6-CN-PiB- positive plaques in $\mathrm{AD}$ (Perez et al., 2015), suggesting that fibrillar deposits of $\mathrm{A} \beta$, rather than its soluble forms, may play a role in the upregulation of proNGF we found in the precuneus. Neurodegeneration is a consequence of $A \beta_{1-40}$ binding to p75 ${ }^{\text {NTR }}$ (Knowles et al., 2009) and CBF perikarya when $A \beta$ oligomers are delivered to the brains of wild type but not p $75^{\text {NTR }}$ deficient mice (Simmons et al., 2014). Interestingly, $\mathrm{CBF}$ degeneration was halted following the depletion of the neurotrophin-binding domain of $\mathrm{p} 75^{\mathrm{NTR}}$ in a mouse model of

\section{$\mathrm{NCl}$}

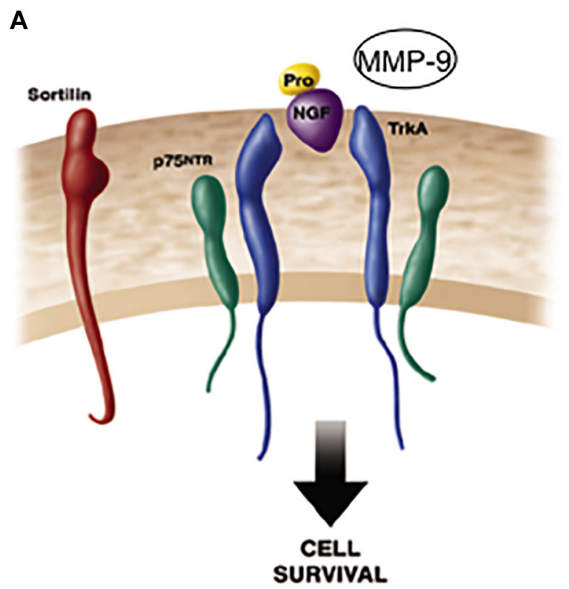

$\mathrm{MCl} / \mathrm{AD}$

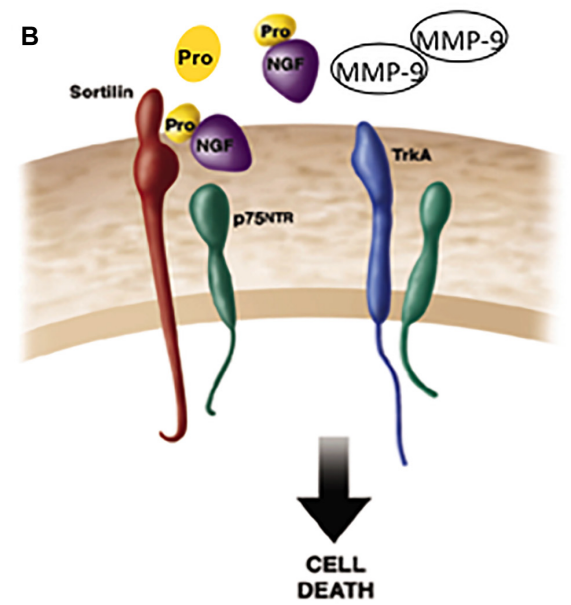

FIGURE 6 | Cartoon demonstrating the shift from cell survival to cell death related to NGF activity during the progression of AD. (A) ProNGF/TrkA complex activates cell survival mechanisms, which is facilitated by the co-expression of p75 NTR on the cell surface in the healthily aged brain. ProNGF also creates a signaling complex by simultaneously binding to sortilin a co-receptor for proNGF. (B) In AD, elevated cortical proNGF in the face of reduced TrkA enhances binding of proNGF to p75 NTR /sortilin complex. Since sortilin acts as a molecular switch governing a p $75^{\mathrm{NTR}}$ mediated pro-apoptotic signal, increased proNGF triggers cell death in the face of decreased TrkA. Modified from Mufson et al. (2012a). 


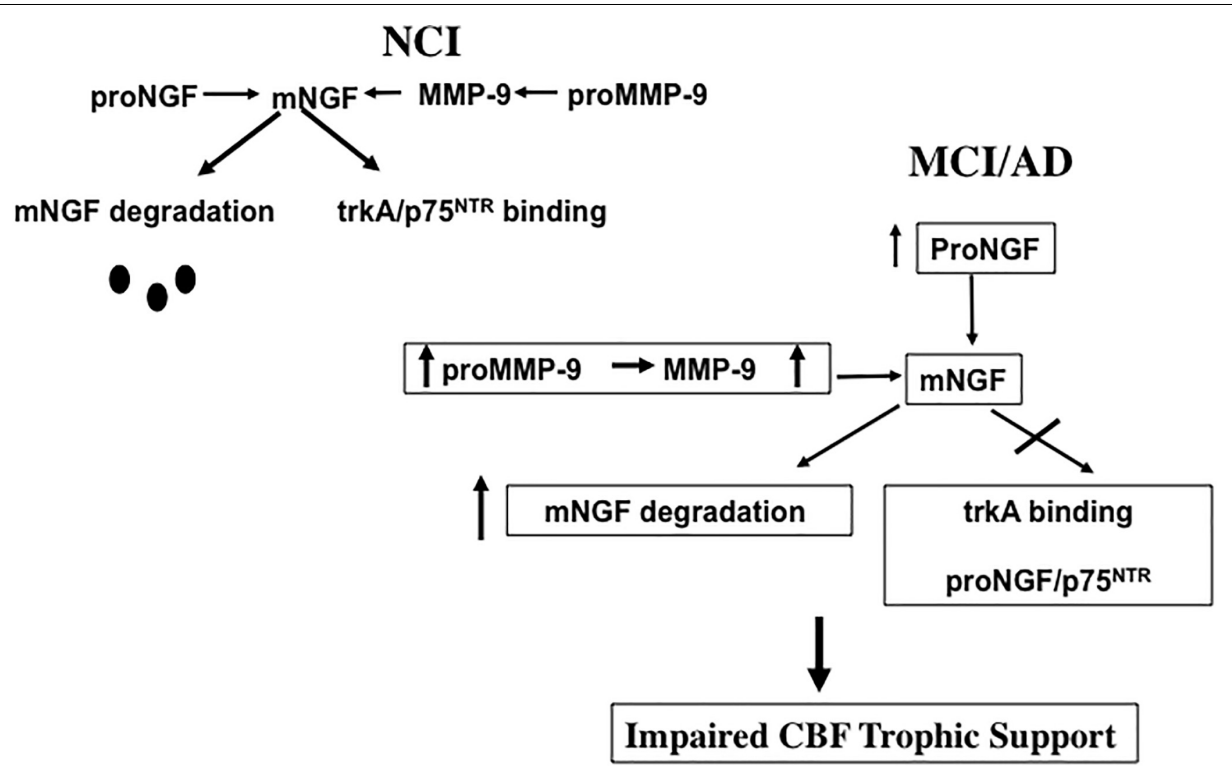

FIGURE 7 | Diagram illustrating the effect of an increase of proMMP-9/MMP-9 has upon the ability of NGF to support cholinotrophic basal forebrain neurons during the progression of $A D$.

$\mathrm{AD}$ (Knowles et al., 2009). These findings suggest that $\mathrm{p} 75^{\mathrm{NTR}}$ signaling is involved in $\mathrm{A} \beta$-induced degeneration and implicate it as an $\mathrm{AD}$ therapeutic target.

\section{HIPPOCAMPAL proNGF AND DOWNSTREAM PATHWAYS DURING THE PROGRESSION OF AD}

The hippocampus is a part of the mesial temporal lobe memory circuit. It develops extensive NFTs but lesser amyloid pathology in the early stages of AD (Hyman et al., 1984, 1990; Braak and Braak, 1991; Arriagada et al., 1992) and receives a major cholinergic input from the medial septal and vertical limb of the diagonal band neurons (Mesulam et al., 1983). Since these septohippocampal cholinergic projection neurons are also dependent upon NGF and its cognate receptors for their survival and degenerate in $\mathrm{AD}$, studies were performed to determine alterations in the hippocampal NGF/proNGF system. Western blot analysis revealed a significant increase in hippocampal proNGF levels in AD but not MCI (Mufson et al., 2012b) in contrast to the neocortex (Bruno et al., 2009; Perez et al., 2015). Of interest is the observation of a significant reduction in TrkA protein levels in MCI hippocampus compared to NCI and $\mathrm{AD}$ and a return to NCI levels during the transition from MCI to AD (Mufson et al., 2012b) (Figure 8). The decrease in TrkA in the face of stable proNGF early in AD may enhance proNGF/p $75^{\mathrm{NTR}} /$ sortilin/NRH2 binding, ultimately shifting the balance from pro-survival to pro-apoptotic signaling in the hippocampus (Figure 8). The upregulation of hippocampal TrkA levels is yet another example of human brain resilience (Mufson et al., 2016a,b) to slow disease progression.
Nerve growth factor and proNGF activate numerous downstream cell survival and apoptotic signaling pathways, respectively (Figure 8). The cell survival protein Erk, which is activated by TrkA phosphorylation, activates nuclear effectors involved in gene transcription (Zhu et al., 2001). Precuneus (Perez et al., 2015) and hippocampal (Mufson et al., 2012b) levels of total Erk, phospho-Erk, and phospho-Erk/Erk ratio are unchanged between $\mathrm{NCI}, \mathrm{MCI}$, and $\mathrm{AD}$. In contrast, stress-activated kinase phospho-JNK, and the ratio of phospho-JNK to JNK were significantly increased in the AD precuneus (Figure 8) (Perez et al., 2015) and hippocampus (Mufson et al., 2012b), whereas total JNK levels were stable similar to the AD hippocampus (Mufson et al., 2012b). Bcl2 a component of the JNK signaling pathway involved in the activation of apoptotic enzymes was upregulated in the precuneus in AD but not MCI (Perez et al., 2015). Of particular interest was the finding that phospho-JNK and the density of AT8 tau-positive NFTs and neuropil threads (NTs) are positively related during the onset of $\mathrm{AD}$, supporting the observation that JNK activation mediates tau phosphorylation at Ser202/Thr205 (AT8 site) (Goedert et al., 1997; Reynolds et al., 1997). Therefore, the activation of JNK pro-apoptotic signaling may play a role in episodic memory impairment in AD.

\section{CHOLINOTROPHIC BASAL FOREBRAIN NEURON GENE EXPRESSION DURING AD PROGRESSION}

The identification of the genetic signature of 'selectively vulnerable' CBF neurons compared to relatively spared neurons during the onset of $\mathrm{AD}$ is crucial for the development of transcriptionally aided drug design to target therapeutics to 

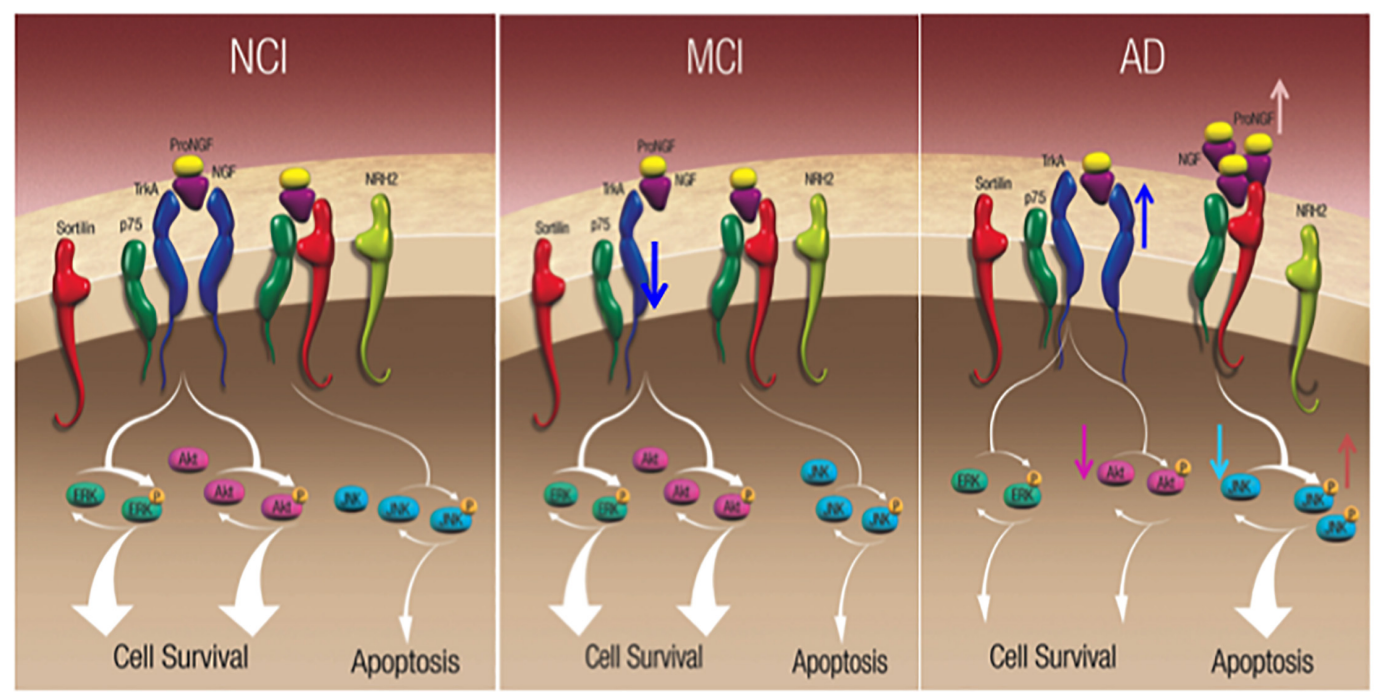

FIGURE 8 | Schematic diagrams showing alteration in hippocampal neurotrophic protein levels during the progression of AD. Reproduced from Mufson et al. (2015),

intervene with the onset of $\mathrm{AD}$. A transcriptionally-driven therapeutic approach may be more likely to preserve brain connectomes including the NGF dependent CBF system that plays a key role in the pathogenesis and onset of dementia, especially during early stages of the disease process (Mufson et al., 2012a). Studies comparing gene expression profiles of CBF neurons identified by $75^{\text {NTR }}$ (Mufson et al., 1989) display a dysregulation of select synaptic-related markers (e.g., downregulation of synaptophysin and synaptotagmin 1 among others), protein phosphatases/kinases (e.g., downregulation of protein phosphatases 1 and 2 subunits and upregulation of cyclin-dependent kinase 5) along with endosomal-lysosomal markers (e.g., upregulation of lysosomal markers cathepsin D, rab4, rab5, and rab7) in MCI and AD compared to age-matched NCI subjects (Ginsberg et al., 2006a,b, 2010; Counts et al., 2011). Moreover, significant downregulation of $\operatorname{Trk} A, \operatorname{TrkB}$, and $\operatorname{Trk} C$ was seen in single CBF neurons microaspirated from the nbM of MCI and AD compared to NCI (Ginsberg et al., 2006b) (Figure 9A), consistent with observations in another vulnerable cell type, hippocampal CA1 pyramidal neurons (Ginsberg et al., 2000, 2010). These findings revealed an intermediate reduction in MCI with the greatest decrement in AD compared to NCI. Moreover, expressed sequence tagged cDNAs (ESTs) [i.e., ESTs targeted to both the extracellular domain (ECD) and tyrosine kinase (TK) domains of Trk receptors] were downregulated. A 'step down' dysregulation of Trk expression, may in part, underlie $\mathrm{CBF}$ neuron demise associated with the clinical presentation of AD. Supporting this concept is the finding that downregulation of $\operatorname{TrkA}$ was associated with several measures of cognitive decline, including the MMSE, a composite global cognitive score (GCS), Episodic, Semantic, Working Memory, Perceptual Speed, and Visuospatial domains as well as Braak NFT stage and neuritic plaque (NP) load within the basal forebrain and hippocampus (Ginsberg et al., 2006b, 2019). Hence, Trk gene expression defects may provide a molecular marker for the transition from MCI to frank AD (Ginsberg et al., 2019). In contrast, p75 ${ }^{\mathrm{NTR}}$ transcript levels were stable in $\mathrm{CBF}$ neurons across the clinical diagnostic groups (Ginsberg et al., 2006b), which was an intriguing finding compared to the significant reduction of $\mathrm{p} 75^{\mathrm{NTR}}$-immunopositive $\mathrm{nbM}$ perikarya in MCI and AD compared to NCI (Gilmor et al., 1999). The discrepancy between $\mathrm{p} 75^{\mathrm{NTR}}$ protein and transcript expression in CBF neurons suggests a disconnection between mRNA transcription and protein translation during disease onset. CBF single population observations in postmortem human brain tissues suggest a relative selectivity in the alteration of the family of cognate NGF receptors during the progression of $\mathrm{AD}$, and that neurotrophic deficits precede or occur during the earliest stages of cognitive decline and neuropathology.

\section{CHOLINOTROPHIC NEURON TAU PATHOLOGY DURING THE PROGRESSION OF AD}

Coincident with altered neurotrophic factor dysfunction during disease progression, CBF neurons also develop intracellular tau inclusions that appear as globose NFTs as well as NTs in MCI and AD (Sassin et al., 2000; Mesulam et al., 2004; Wu et al., 2005; Vana et al., 2011). The human brain contains three isoforms of tau with three tandem repeats (3Rtau; Mapt1, Mapt3, and Mapt5) and three tau isoforms with four tandem repeats (4Rtau; Mapt2, Mapt4, and Mapt6). Custom-designed microarray evaluation of CBF neurons did not reveal changes in any of the six tau transcripts between AD, MCI and NCI subjects (Ginsberg et al., 2006a). However, a significant shift in the ratio of 3 Rtau/4Rtau ratio was observed with a decrease in 3Rtau expression relative to 4Rtau levels for all tau transcripts. Tau transcript data suggest a fluctuation 
A

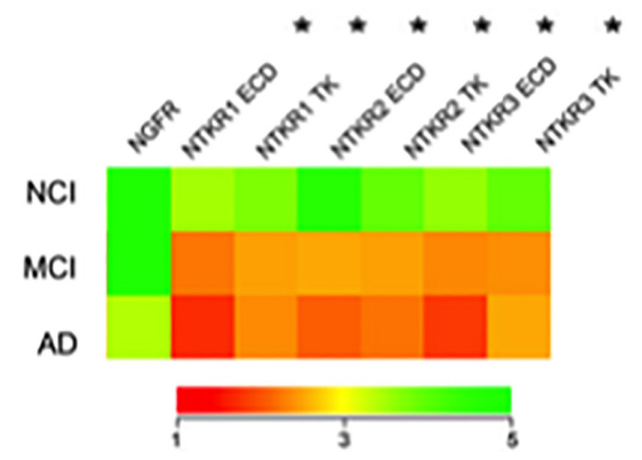

B

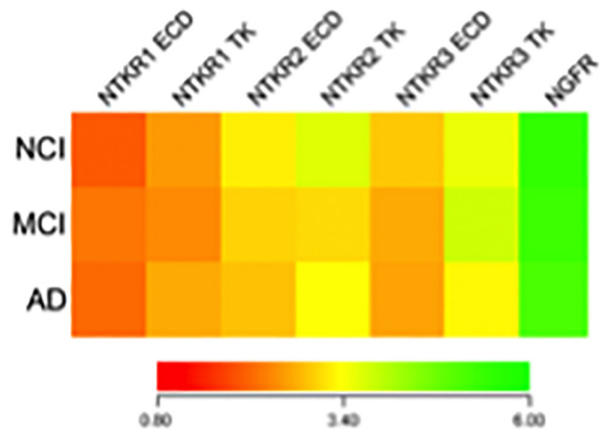

C

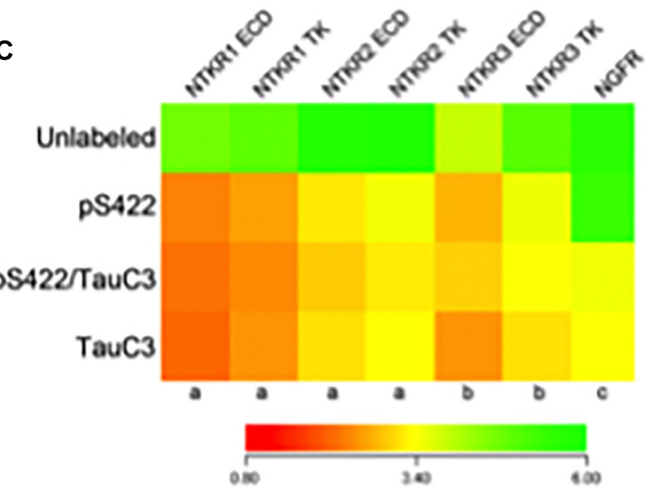

FIGURE 9 | Findings derived from single cholinotrophic neuron profiling during the progression of AD. (A) Heatmap demonstrating downregulation of Ntrk1 (TrkA), Ntrk2 (TrkB), and Ntrk3 (TrkC) \{both the extracellular domain (ECD) and the tyrosine kinase domain (TK)\} (asterisk), but not Ngfr (p75 ${ }^{\mathrm{NTR}}$ ) during the progression of dementia. Trk gene expression in $\mathrm{MCl}$ is intermediate to $\mathrm{AD}$, indicating a step-down effect from $\mathrm{NCl}$ to $\mathrm{MCl}$ to $\mathrm{AD}$. (B) Heatmap of the relative expression profiles for select neurotrophin transcripts in pS422+ nbM neurons. No differences in Trk receptors or Ngfr were found in $\mathrm{MCl}$ or $\mathrm{AD}$ compared to NCl. (C) Downregulation of Trk receptors was found in pS422+ $\mathrm{nbM}$ neurons compared to unlabeled control neurons. Downregulation of Ngfr was associated with the neoepitope TauC3. Key: (a) unlabeled > pS422, $p<0.001$; (b) unlabeled > pS422, $p<0.01$; (c) pS422 > pS422+/TauC3+, $p<0.01$. (A) Adapted with permission from Ginsberg et al. (2006b). (B,C) Adapted with permission from Tiernan et al. (2018a).

in gene dosage for 3Rtau and 4Rtau within CBF neurons in $\mathrm{MCI}$ and $\mathrm{AD}$, which was not seen in normal aging (Ginsberg et al., 2006a).
Single neuron expression profiling investigations have addressed the extent to which levels of transcripts encoding neurotrophin receptors are altered in individual nbM neurons labeled for the pretangle marker pS422+, the late stage caspase-cleaved tau marker TauC3+ or pS422/TauC3+ compared to unlabeled neurons obtained from NCI, MCI, and AD cases provided by the RROS (Tiernan et al., 2018a). Quantitative analyses compared transcript signal intensities between clinical stages or between tau neuronal phenotypes. Comparison of transcript expression in pS422+ nbM neurons microaspirated from each clinical stage revealed no statistical differences (Figure 9B). However, when analyzed independent of clinical diagnosis, expression levels of key genes regulating neurotrophin receptor expression were altered as classified by a phenotypic transition from unlabeled to $\mathrm{pS} 422+$ to pS422+/TauC3+ to TauC3+ in nbM neurons (Figure 9C). Compared to unlabeled, pS422+ nbM neurons showed a significant downregulation of six mRNAs encoding the intracellular TK and extracellular ECD domains of the neurotrophin receptors TrkA (Ntrk1 TK, 50\% downregulation; Ntrk1 ECD, 53\%), TrkB (Ntrk2 TK, 45\%; Ntrk2 ECD, 42\%), and TrkC (Ntrk3 TK, 38\%; Ntrk3 ECD, 35\%) (Figure 9C). In addition, we found that these same transcripts are significantly downregulated in neurons containing the early pretangle tau antibody Tau Oligomeric Complex 1 (TOC1) (Counts, unpublished observations), lending support to the hypothesis that neurotrophic dysfunction occurs before frank NFT formation.

In contrast, transcript levels of the mRNA encoding the pan-neurotrophin receptor $\mathrm{p} 75^{\mathrm{NTR}}(\mathrm{Ngfr})$ were not decreased until the appearance of TauC3. This expression data complements our stereologic finding demonstrating that TauC3 and $\mathrm{p} 75^{\mathrm{NTR}}$ did not co-localize within the CBF at the protein level (Mufson et al., 2002b; Vana et al., 2011). Prior studies reported that Trk receptors expression levels are downregulated in nbM neurons in MCI and AD relative to NCI (Ginsberg et al., 2006b). On the other hand, it was found that Ntrk transcripts were downregulated by the phenotypic transition from nbM non-labeled to pS422+ neurons, whereas no difference was found in Ntrk expression in pS422+ neurons from NCI, MCI, and AD cases (Figure 9C).

We previously performed antibody imunostaining for TOC1 (Patterson et al., 2011; Ward et al., 2013) and $75^{\mathrm{NTR}}$ to quantify pretangle tau oligomeric assemblies, which most likely are the more neurotoxic species of tau (Berger et al., 2007; Maeda et al., 2007; Kopeikina et al., 2011; Lasagna-Reeves et al., 2012; Sahara et al., 2013) within CBF neurons during the progression of AD (Tiernan et al., 2018b). Here the number of p $75^{\mathrm{NTR}}+/ \mathrm{TOC} 1+\mathrm{nbM}$ neurons progressively increased from NCI to MCI to AD, whereas single TOC1+ nbM neurons were lower in NCI and MCI but increased in AD. A subanalysis of $\mathrm{p} 5^{\mathrm{NTR}}+, \mathrm{p} 75^{\mathrm{NTR}}+/ \mathrm{TOC} 1+$, and $\mathrm{TOC} 1+\mathrm{nbM}$ neurons in NCI cases with a low Braak score (Stages III) compared to a high Braak score (Stages III-V) revealed a significant increase in the number of $\mathrm{p}^{\mathrm{NTR}}+/ \mathrm{TOC} 1+$ dual-immunolabeled neurons in NCI-high pathology compared to NCI-low pathology cases. The reduction of $\mathrm{p}^{75^{\mathrm{NTR}}+}$ 
nbM neurons was associated with poorer GCS and MMSE performance test scores. TOC1 primarily co-localized with pS422 in NCI, but the transition to MCI and AD was marked by a shift from TOC1+/pS422+ toward triple-labeled TOC1+/pS422+/MN423+ neurons, implying a specific, linear order of epitope occurrence in nbM cholinotrophic neurons during disease progression. This arrangement suggests that aberrant phosphorylation primes the tau protein toward additional phosphorylation and conformational events (LunaMunoz et al., 2007; Bertrand et al., 2010) that facilitates oligomerization (Iqbal et al., 2013). Given the findings that prefibrillar tau pathology is related with molecular and cellular alterations within nbM neurons (Tiernan et al., 2016, 2018b), the appearance of prefibrillar oligomeric tau likely precedes cell loss. Evidence implicating tau pathology as a driver of neurotrophic dysfunction is seen in tau transgenic mice and tau-transfected neuronotypic cells, which display a downregulation of the trophic substance brain-derived neurotrophic factor (BDNF) (Rosa et al., 2016). On the other hand, NGF regulates tau turnover (Sadot et al., 1996) and post-translational modifications including phosphorylation, cleavage, and ubiquitination (Nuydens et al., 1997; Shelton and Johnson, 2001; Babu et al., 2005; Amadoro et al., 2011), suggesting that neurotrophic abnormalities initiate tau pathology within CBF neurons (Canu et al., 2017). Moreover, the reduced microtubule-binding capacity of tau and/or the somatodendritic accumulation of tau may contribute to axonal degeneration and associated NGF/TrkA signaling dysregulation (Mufson et al., 2002a; Schindowski et al., 2008). Additionally, proNGF induces tau hyperphosphorylation in vitro via the enhanced activity of GSK3 $\beta$ (Shen et al., 2018). Based on these collective results future analysis of the potential interactions between aberrant tau metabolism and disrupted neurotrophic signaling in cholinotrophic nbM neurons (Capsoni et al., 2000).

\section{CHOLINOTROPIC EPIGENETIC ALTERATIONS DURING THE PROGRESSION OF AD}

Histone acetylation and deacetylation are also involved in $\mathrm{CBF}$ neuron function via their regulation of ChAT (Aizawa and Yamamuro, 2010; Aizawa et al., 2012; Bekdash, 2016) indicating a potential role for epigenetics in neuronal selective vulnerability in AD. Evidence is growing that histone deacetylases (HDACs), epigenetic enzymes with deacetylase activity, located within the nucleus and cytoplasm of neurons play a role in AD pathogenesis (Ding et al., 2008; Guan et al., 2009; Xu et al., 2011; Cook et al., 2012; Graff et al., 2012). Several HDACs are related with cellular events dysfunctional in $\mathrm{AD}$, including endoplasmic reticulum stress (HDAC4) (Shen et al., 2016), autophagy (HDAC6) (Pandey et al., 2007), mitochondrial transport (HDAC6) (Chen et al., 2010), tau hyperphosphorylation (HDAC6) (Ding et al., 2008) and $A \beta$ and tau accumulation (SIRT1) (Julien et al., 2009; Lalla and Donmez, 2013). However, whether epigenetic dysregulation occurs in cholinotrophic nbM neurons remains under-investigated in $\mathrm{AD}$.
Of the HDACs, HDAC2 has received extensive investigation due to a role in the modulation of transcripts involved in cognition via chromatin plasticity regulation (Dawson and Kouzarides, 2012; Graff and Tsai, 2013; Volmar and Claes, 2015). In this regard, a clinicopathological investigation revealed alterations in HDAC2-immunoreactive (ir) nuclei within nbM neurons during the onset of AD (Mahady et al., 2019). This study revealed that normally rounded HDAC2-ir nbM nuclei appeared ovoid, flattened, and eccentrically located within the soma in MCI, mild AD (mAD) and severe AD (sAD) (Figures 10A-H). HDAC2 nuclear intensity was significantly reduced in $\mathrm{SAD}$ compared to the other clinical groups examined (Figures 10D,H). Moreover, HDAC2-ir nuclear intensity was significantly reduced in $\mathrm{mAD}$ compared to the NCI and MCI groups (Figure 10I). This study further demonstrated that HDAC2 nbM nuclear intensity was not significantly different in NCI and MCI. The sAD nuclear area was significantly smaller than observed in NCI, MCI, and mAD (Figure 10J). In mAD, HDAC2-ir nuclei displayed significantly smaller area compared to NCI. HDAC2-ir nuclear intensity was found to correlate with working memory and a GCS (Mahady et al., 2019). A decline in the number of nbM p75 ${ }^{\text {NTR }}$ immunoreactive neurons decreased across disease stages and was related to a reduction in HDAC2 nuclear immunoreactivity (Mahady et al., 2019). Similarly, a reduction in $\mathrm{HDAC} 2$ nuclear immunoreactivity was inversely related to an increase in the number of AT8 pretangle-bearing nbM neurons. Quantitation of the intensity of HDAC2 nuclei revealed a significant reduction in non-tangle bearing $\mathrm{p} 75^{\mathrm{NTR}}$ positive neurons in $\mathrm{mAD}$ and $\mathrm{sAD}$ compared to $\mathrm{NCI}$ and MCI. NbM neurons triple-labeled for $\mathrm{p} 75^{\mathrm{NTR}}$, the pretangle maker AT8 or late-stage tau epitope, TauC3, displayed an even larger decrease in HDAC2 immunoreactivity in AD compared to non-tangle bearing $\mathrm{p} 75^{\mathrm{NTR}}$ neurons at each disease stage (Mahady et al., 2019). Within-group analysis indicated HDAC2ir was highest in non-tangle bearing cholinergic perikarya in each clinical group (Mahady et al., 2019). Interestingly, HDAC2 nuclear immunoreactivity was further decreased in HDAC2/AT8/Thioflavin-S or HDAC2/TauC3/Thioflavin-S neurons in $\mathrm{MCI}$ and $\mathrm{mAD}$. These findings suggest that a reduction in HDAC2 expression occurs before the onset of fibrillar tau pathology and that this alteration is exacerbated by phosphorylated and conformational tau epitopes in nbM neurons during the progression of AD. Although ChAT mRNA expression and protein levels are epigenetically modulated by hyperacetylation of the core promoter region of the ChaT transcript (Aizawa et al., 2012), decreased HDAC2 nuclear levels were found within cholinergic nbM neurons in MCI, but significantly decreased ChAT nbM protein levels were seen only in AD (Mahady et al., 2019). These phenomena suggest that HDAC2 nuclear protein downregulation does not alter neuronal ChAT activity early in the disease process. Immunohistochemical analysis of HDACs in cortical cholinergic nbM projection sites demonstrated differential regional findings. For instance, although HDAC1 and HDAC2 are reduced in AD entorhinal cortex (Mastroeni et al., 2010), HDAC2, but not HDAC1 or HDAC3, are increased in hippocampal and entorhinal cortex neurons in $\mathrm{AD}$ compared to HDAC2 in control 


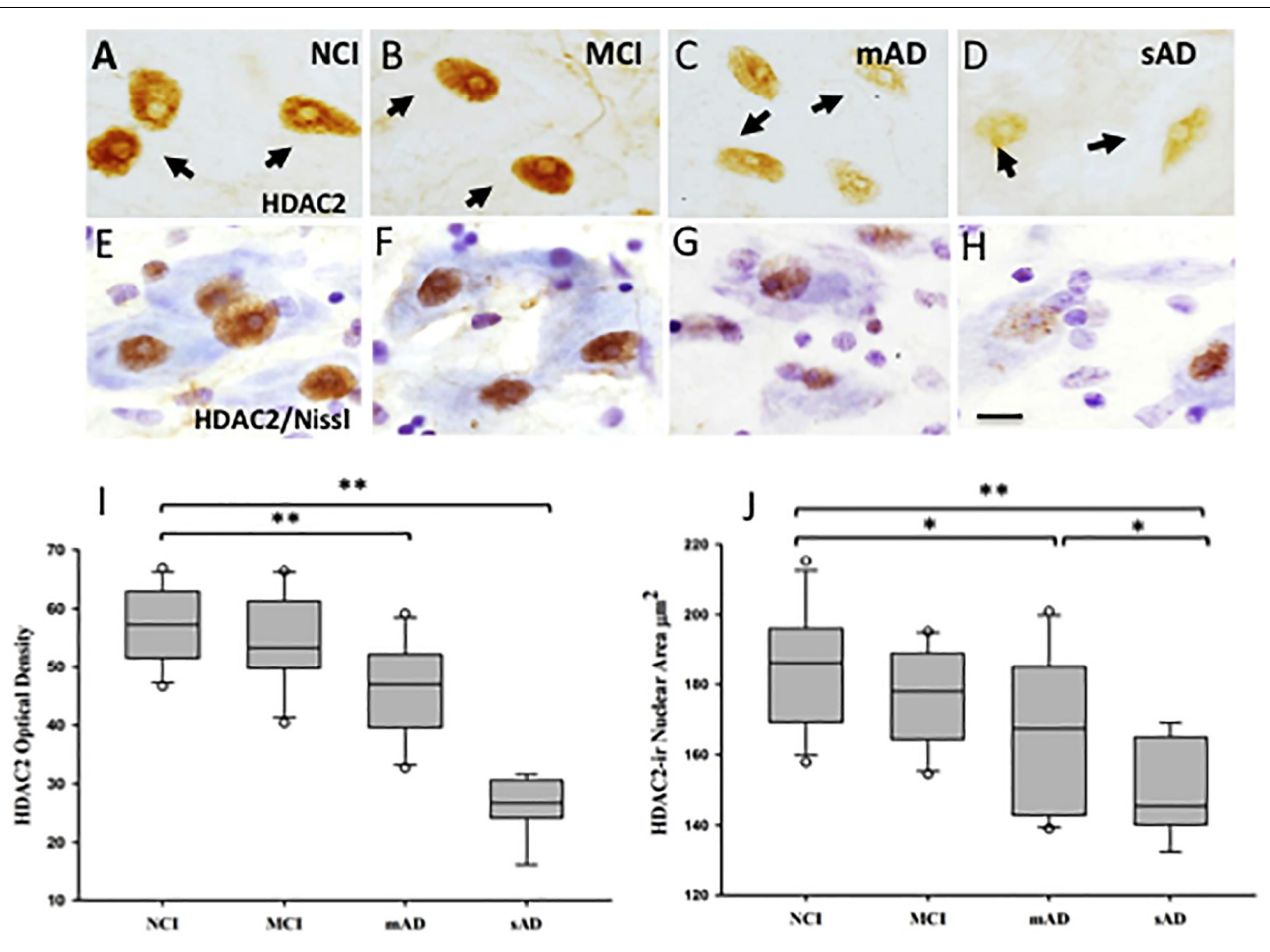

FIGURE 10 | Photomicrographs of single HDAC2 (A-D) and HDAC2 and cresyl violet dual stained (E-H) nucleus basalis neurons. Nuclei positive for HDAC2 lost their rounded shape and were displaced to the periphery of the soma in the MCl, mAD, and SAD groups. (I) Box plots showing HDAC2-ir was significantly decreased in mAD compared to $\mathrm{NCl}$ and $\mathrm{MCl}(p<0.001)$, and SAD compared to NCl, $\mathrm{MCl}$, and mAD ( $p<0.001)$. (J) Box plots showing area of HDAC2-ir nuclei was significantly decreased in mAD compared to $\mathrm{NCl}(p=0.03)$ and in SAD compared to $\mathrm{NCl}(p<0.001)$, MCl $(p<0.001)$, and mAD $(p=0.04)$ groups. Circles in box plots indicate outliers. ${ }^{*} p<0.05$; ${ }^{* *} p<0.001$. Scale bar: $10 \mu \mathrm{m}$. Reproduced from Mahady et al. (2019).

subjects (Graff et al., 2012). Western blots of frontal cortex revealed significant increases in $\mathrm{HDAC} 1, \mathrm{HDAC} 3, \mathrm{HDAC} 4$, and HDAC6 in MCI and mAD compared to NCI whereas HDAC2 levels remained stable (Mahady et al., 2018). These findings suggest that differential epigenetic regulation occurs across brain regions affected in $\mathrm{AD}$.

\section{CHOLINOTROPHIC BIOMARKERS FOR THE PROGRESSION OF AD}

As discussed above, protein levels of proNGF are increased in postmortem neocortex (Peng et al., 2004) and hippocampus (Mufson et al., 2012b) of subjects who died with a clinical diagnosis of MCI or mild AD compared to NCI, respectively, which correlated with poorer antemortem cognitive test scores (Peng et al., 2004; Mufson et al., 2012b). These observations initiated an investigation of whether altered CSF levels of proNGF mark a transition from NCI to MCI and AD. Ventricular CSF (vCSF) was obtained postmortem from RROS participants and premortem lumbar CSF was collected from subjects clinically diagnosed as CDR 0 (no dementia), CDR 0.5 (MCI or very mild AD), or CDR 1 (mild AD) at the Washington University Knight AD Research Center (Counts et al., 2016). Quantitative western blotting of vCSF revealed a significant (50\%) increase in proNGF levels in aMCI compared to NCI and a $70 \%$ increase in AD compared to NCI, which displayed a significant inverse relationship between increasing vCSF proNGF levels and cognitive deterioration (Counts et al., 2016). Lumbar CSF proNGF levels were significantly (30\%) increased in CDR 0.5 and CDR 1 compared to CDR 0 cases. Although no difference in levels of $A \beta_{1-42}$, total tau, phospho-tau, or phospho-tau/A $\beta_{1-42}$ was found between groups, the ratio of total tau $/ A \beta_{1-42}$ levels was $50 \%$ higher in CDR 1 compared to CDR 0 subjects. Ratios were calculated for proNGF/A $\beta_{1-42}$, proNGF/total tau, and proNGF/phospho-tau to determine if the inclusion of CSF proNGF levels improved the reliability of these biomarkers. Interestingly, proNGF/A $\beta_{1-42}$ levels were $50 \%$ higher in $\mathrm{CDR}$ 0.5 and CDR 1 compared to CDR 0, whereas proNGF/total tau and proNGF/phospho-tau were unchanged between groups, suggesting the inclusion of proNGF as a candidate biomarker will improve the diagnostic probability needed to identify people in the preclinical or prodromal stages of AD.

\section{NGF THERAPY AS A TREATMENT STRATEGY FOR AD}

Evidence derived from studies employing NGF as a treatment strategy to rescue the cholinotrophic cortical projection system has revealed some promising results regarding prevention of $\mathrm{CBF}$ neuron atrophy and a correction of behavioral deficits 
resulting from experimental damage or normal aging (Hefti, 1986; Williams et al., 1986; Hartikka and Hefti, 1988; Hatanaka et al., 1988; Nabeshima et al., 1994; Burgos et al., 1995; Charles et al., 1996; Humpel and Weis, 2002). This evidence led to the concept that treatments that facilitate NGF would be beneficial in reversing cholinotrophic dysfunction in AD. However, examination of studies of early NGF systemic administration showed several weaknesses including bioavailability of the neurotrophin to reach target neurons, unregulated neurotransmitter release, hyperinnervation, sprouting of neurons, sympathetic stimulation, induction of antibodies, cachexia, and hyperalgesia (Sramek and Cutler, 1999; Jonhagen, 2000; McArthur et al., 2000; Apfel, 2001). However, after further testing in rat and non-human primate animal models (Gnahn et al., 1983; Hefti, 1986; Gage et al., 1989, 1990; Hefti et al., 1989; Higgins et al., 1989; Tuszynski et al., 1990, 1991, 1996; Blesch and Tuszynski, 1995) and taking into account past failures (e.g., poor drug delivery and unwanted systemic side effects), a Phase I clinical trial was undertaken to determine the utility of ex vivo NGF gene therapy for AD (Tuszynski et al., 2005). The goal was both to protect cholinotrophic neurons within the nbM from degeneration as well as augment the function of remaining cholinergic neurons by intracranial delivery of human NGF. During clinical trials, patients with AD, underwent NGF transcript therapy using ex vivo or in vivo gene transfer directed at the cholinergic neurons within the nbM. Degenerating nbM neurons were found to respond to NGF, with axonal sprouting toward the NGF source (Figure 11). Participants that had unilateral gene transfer displayed neuronal hypertrophy in the NGF-treated cholinergic nbM.

Moreover, patients that sustained adeno-associated viral vector (serotype 2)-mediated NGF gene transfer, displayed activation of cellular signaling and functional markers. Interestingly, nbM neurons that exhibited pathologic tau and those that were tau immunonegative both expressed NGF, indicating that tangle-bearing neurons can be infected with therapeutic genes, which activate cell signaling. No adverse effects related to NGF treatment were found in these studies. In summary, these findings revealed that degenerating neurons can respond to NGF with axonal sprouting, cell hypertrophy, and activation of functional markers. These studies demonstrated that NGF-induced sprouting persisted for 10 years post NGF gene transfer and that this therapy appears safe over long periods of time (Tuszynski et al., 2015). It should be kept in mind that these studies were not double-blind, placebo-controlled clinical trials. Further clinical investigation of gene therapy approaches is warranted.

\section{SMALL MOLECULE NEUROTROPHIN COMPOUNDS FOR TREATMENT OF AD}

Small molecule partial agonist and antagonist activators of NGF receptors have been considered for the treatment of AD (Skaper, 2008). For example, a high-throughput screening assay of small-molecule agonists for TrkA identified gambogic amide, an alkaloid used in traditional Chinese medicine as a possible candidate (Jang et al., 2007). Gambogic amide binds selectively to TrkA (but not TrkB and TrkC), phosphorylates TrkA tyrosine residues, and activates the Akt and Erk TrkA-mediated NGF signaling pathways. Gambogic amide has been demonstrated to ameliorate excitotoxic damage and promote neurite outgrowth in PC12 cells and reduce kainic acid neuronal induced cell death in mice (Jang et al., 2007).

Several lines of evidence also point to the modulation of degenerative signaling promoted by $\mathrm{p} 75^{\mathrm{NTR}}$ as a potential therapeutic target for AD. Through either its constitutive activity in the unliganded state, or via that stimulated by its proneurotropin (proNT) ligands, $\mathrm{p} 75^{\mathrm{NTR}}$ promotes degenerative signaling mechanisms including activation of JNK, caspase, and RhoA (Casaccia-Bonnefil et al., 1996; Harrington et al., 2002; Troy et al., 2002; Ibanez and Simi, 2012; Coulson and Nykjaer, 2013) each of which likely contributes to AD-related degeneration (Figure 12). Crossing various $\mathrm{AD}$ mouse models with a $\mathrm{p} 75^{\mathrm{NTR}}$ knockout mouse construct results in reduced neuronal degeneration (Sotthibundhu et al., 2008; Knowles et al., 2009; Murphy et al., 2015). Multiple studies have identified genetic polymorphisms in the genes encoding $\mathrm{p} 75^{\mathrm{NTR}}$, proNGF, proBDNF, or $\mathrm{p} 75^{\mathrm{NTR}}$ co-receptors including sortilin and SorCS2 that mediate proNT binding associated with increased AD risk (Cozza et al., 2008; Di Maria et al., 2012; Anastasia et al., 2013; Reitz et al., 2013; Andersson et al., 2016; Matyi et al., 2017). The first small molecule ligands found to interact with $\mathrm{p} 75^{\mathrm{NTR}}$ and modulate its signaling were identified using an in silico screening strategy based on synthetic peptides modeled on the loop I domain of NGF and mutational analyses of neurotrophin ligands (Massa et al., 2006). Two prototype small molecule compounds, LM11A-31 and LM11A-24, were found to prevent neuronal
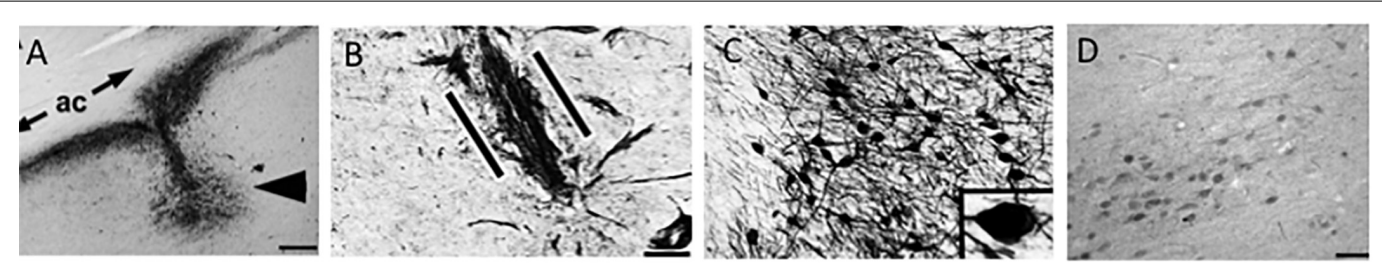

FIGURE 11 | (A) Nerve growth factor (NGF) immunolabeling shows the site of NGF gene delivery in the human nucleus basalis of Meynert (arrowhead) under the anterior commissure (ac). Scale bar $=325 \mu \mathrm{m}$. The patient received the injection 3 years previously. (B) p75 NTR neurotrophin receptor immunolabeling shows basal forebrain cholinergic axons penetrating into the graft (between the parallel lines) in a linear fashion. Scale bar $=25 \mu \mathrm{m}$. (C) Nerve growth-factor-expressing neurons compared with less intense labeling in the nucleus basalis of Meynert neurons located $3 \mathrm{~mm}$ from the injection site (D). Scale bar in (C) = $100 \mu \mathrm{m}$ and in (D) = $325 \mu \mathrm{m}$. Images reproduced from Tuszynski et al. (2015). 
$A \beta$-driven degenerative mechanisms inhibited by $p 75$ receptor modulator LM11A-31

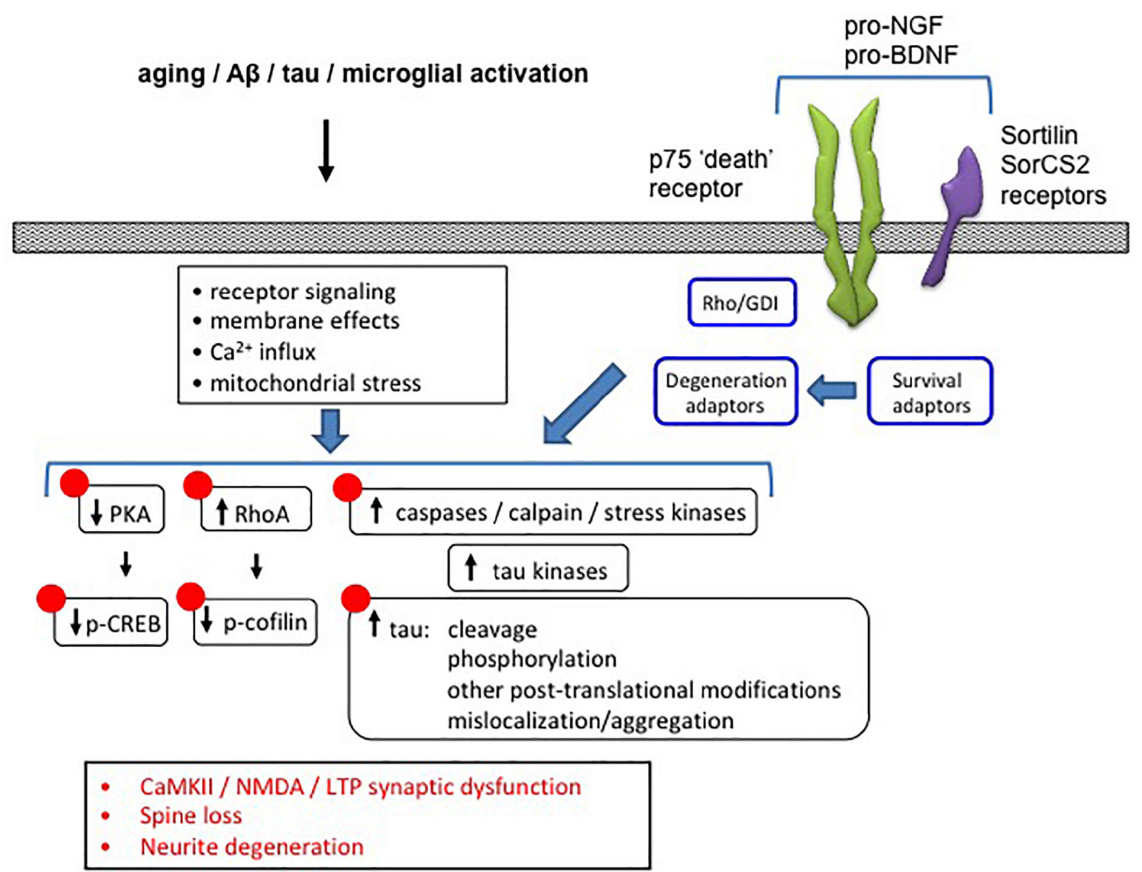

FIGURE 12 | Diagram showing the association between $\mathrm{p} 75^{\mathrm{NTR}}$ and Alzheimer's-related degenerative signaling networks. Aging, A $\beta$ and other processes produce numerous changes in signaling pathways involved in neurite and synapse function and degeneration. Degenerative signaling promoted by $\mathrm{p} 75^{\mathrm{NTR}}$ in either its unliganded state, or in response to proneurotrophin binding to $\mathrm{p} 75^{\mathrm{NTR}}$ and its sortilin family co-receptors, and mediated through one or more receptor adaptors enables or promotes AD-related degenerative processes. LM11A-31, which interferes with proneurotrophin degenerative signaling and promotes supportive/survival signaling through $p 75^{\mathrm{NTR}}$, reverses many of these AD-associated effects (indicated by $\bullet$ ).

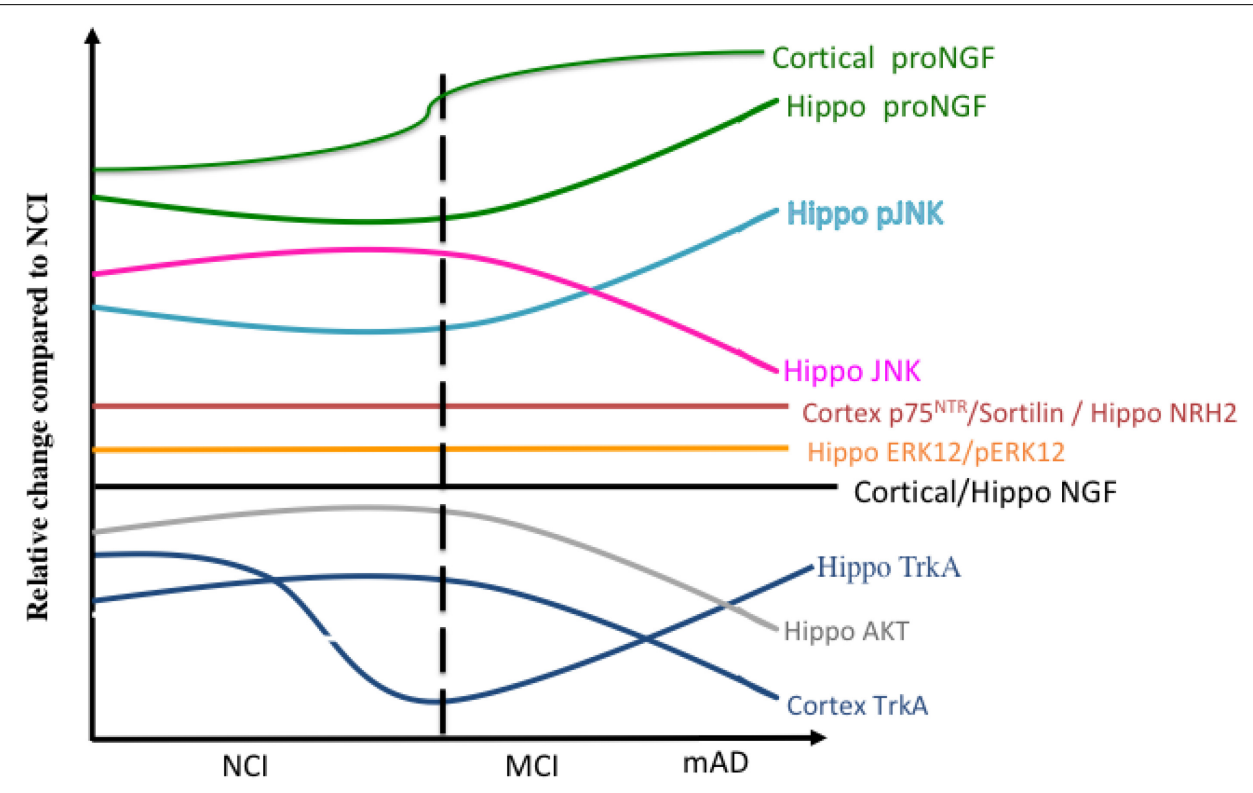

FIGURE 13 | Summary diagram showing the changes in the relative levels of NGF-related proteins in the cortex and hippocampus during the progression of AD. 
death in cell culture conditions under which neuronal survival is dependent on the addition of neurotrophins. These small molecule ligands effectively acted as positive $\mathrm{p} 75^{\mathrm{NTR}}$ modulators, inhibited proNGF-induced cell death (Massa et al., 2006) and blocked binding of proNGF to p75 ${ }^{\text {NTR }}$ (Tep et al., 2013). Under these conditions, small molecule ligands could be described as p75 ${ }^{\text {NTR }}$ 'antagonists.' LM11A-31 has been shown to stimulate recruitment of interleukin-1 receptor-associated kinase (IRAK) survival adaptor to $\mathrm{p} 75^{\mathrm{NTR}}$ and to upregulate downstream $\mathrm{NF}-\kappa \mathrm{B}$ and Akt pro-survival signaling. Neurotrophic activity and signaling were absent in cultures using $\mathrm{p} 75^{\mathrm{NTR}}-/-$ neurons or in the presence of $\mathrm{p} 75^{\mathrm{NTR}}$-ECD blocking antibody (Massa et al., 2006). LM11A-31 blocks proNGF-induced degenerative mechanisms in models of spinal cord injury (Tep et al., 2013), neurogenic bladder dysfunction (Ryu et al., 2018), and arthritis (Minnone et al., 2017).

Multiple preclinical studies indicate a role for small molecule modulation of $\mathrm{p} 75^{\mathrm{NTR}}$ as a therapeutic approach for slowing progression of $\mathrm{AD}$-related degenerative mechanisms. Intracellular signaling networks linked to $\mathrm{p} 75^{\mathrm{NTR}}$ have substantial integration with degenerative signaling networks implicated in AD (Nguyen et al., 2014). In a model of AD in which cultured hippocampal neurons are exposed to $A \beta$ oligomers, LM11A-31 and LM11A-24 inhibited the following $A \beta$-induced degenerative mechanisms: activation of calpain/cdk5, GSK3 $\beta$, JNK c-Jun, p38 kinase, RhoA; excessive tau phosphorylation; and inactivation of Akt and CREB (Yang et al., 2008). These ligands also blocked $\mathrm{A} \beta$-induced neuritic dystrophy and hippocampal long-term potentiation (LTP) impairment. In studies employing the hAPP ${ }^{\text {Lond/Swe }}$ AD mouse model, a once-daily administration of LM11A-31 over 3 months corrected behavioral deficits and inhibited neurodegenerative molecular and cellular pathology including tau phosphorylation and misfolding, neurite dystrophy, microglial activation and astrocyte activation (Knowles et al., 2013; Nguyen et al., 2014). However, there was no effect on lowering soluble $A \beta$ or amyloid plaque levels, consistent with a mechanism in which modulation of $\mathrm{p} 75^{\mathrm{NTR}}$ inhibits the ability of $A \beta$ to promote neural degeneration including tau-related molecular degenerative events and synaptic failure. The ability of LM11A-31 to reduce measures of microglial activation in hAPPLond/Swe $\mathrm{AD}$ mice was confirmed using multiple markers of microglial activation as well as micro-PET imaging using a PET ligand directed to the translocator protein (TSPO) (James et al., 2017). In hAPP ${ }^{\text {Lond/Swe }}$ AD mouse studies employing treatment in late-stage, application of LM11A-31 resulted in a partial reversal of neural degeneration, perhaps indicative of a particularly robust biological effect (Simmons et al., 2014). Figure 12 summarizes the ability of the p $75^{\text {NTR }}$ modulator LM11A-31, which interferes with proNT degenerative signaling and promotes supportive/survival signaling through p $75^{\mathrm{NTR}}$, to inhibit/reverse AD degenerative mechanisms.

Based on in vivo preclinical studies, a modified formulation of LM11A-31, as a first-in-class compound directed against a novel target, was tested in a phase 1 trial in normal subjects and found to be safe. This compound is currently in a randomized, double-blinded, phase $2 \mathrm{a}$ exploratory endpoint trial in subjects with mild to moderate AD (NCT03069014). Treatment is administered via daily oral capsules for 6 months, and measurements at baseline and post-treatment include the following: cognitive testing with multiple batteries; MRI volumetric measures and FDG-PET imaging; and CSF AD core biomarkers along with biomarkers relevant to target engagement and mechanisms of action.

\section{CONCLUDING COMMENTS}

Figure 13 summarizes the relative changes of NGF upstream and downstream signaling pathways within the cortex and hippocampus during the progression of $\mathrm{AD}$. A preponderance of data indicates that normative levels of NGF and its cognate receptors are required for the survival and maintenance of the cholinotrophic system. The preservation of cholinergic nbM neurons in the face of reduced numbers of TrkA and $\mathrm{p} 75^{\mathrm{NTR}}$-positive neurons in MCI and mild AD indicates that there is not a frank loss of cholinergic perikarya perse but a phenotypic downregulation of receptor proteins early in the disease process. Both transcript and protein data indicate that $\mathrm{CBF}$ neuron dysfunction is associated with an imbalance between TrkA-mediated survival signaling and proNGF/p $75^{\mathrm{NTR}}$-mediated pro-apoptotic signaling. Despite these degenerative events, the cholinotrophic system is capable of cellular resilience and/or neuroplasticity during the prodromal (DeKosky et al., 2002) and even the later stages of the disease (Mufson et al., 2012b). In addition to neurotrophic dysfunction, alterations in nuclear epigenetic proteins occur within cholinotrophic nbM neurons during the progression of $\mathrm{AD}$, particularly HDAC2, suggesting a mechanism associated with changes in transcript expression. Increased proNGF quantified in postmortem vCSF or premortem lumbar CSF marked the transition to $\mathrm{MCI}$ and to $\mathrm{AD}$, suggesting that this proneurotrophin is a useful biomarker of disease progression. Clinical trials provide evidence that NGF gene therapy has the potential to be a treatment approach for the prevention of $\mathrm{CBF}$ degeneration in AD. Perhaps combining this therapeutic approach with the development of small molecule agonists to TrkA to facilitate prosurvival signaling (Jang et al., 2007) or small molecule antagonists to $\mathrm{p} 75^{\mathrm{NTR}}$ for anti-apoptotic actions should be added to the treatment toolbox for MCI and AD.

\section{AUTHOR CONTRIBUTIONS}

EJM designed and wrote the manuscript. SEC, SDG, LM, SEP, SMM, FML, and MDI contributed text and editing of the manuscript.

\section{FUNDING}

Funded by grants PO1 AG014449, RO1 AG043375, PO1 AG107617, and P30AG053760 from the National Institutes of Health, the Alzheimer's Association, and Barrow and Beyond at the Barrow Neurological Institute. We are indebted to the Catholic nuns, priests, and lay brothers who participated in the Rush Religious Orders Study. 


\section{REFERENCES}

Aizawa, S., Teramoto, K., and Yamamuro, Y. (2012). Histone deacetylase 9 as a negative regulator for choline acetyltransferase gene in NG108-15 neuronal cells. Neuroscience 205, 63-72. doi: 10.1016/j.neuroscience.2011.12.024

Aizawa, S., and Yamamuro, Y. (2010). Involvement of histone acetylation in the regulation of choline acetyltransferase gene in NG108-15 neuronal cells. Neurochem. Int. 56, 627-633. doi: 10.1016/j.neuint.2010.01.007

Allen, S. J., MacGowan, S. H., Treanor, J. J. S., Feeney, R., Wilcock, G. K., and Dawbarn, D. (1991). Normal B-NGF content in Alzheimer's disease cerebral cortex and hippocampus. Neurosci. Lett. 131, 135-139. doi: 10.1016/03043940(91)90354-v

Al-Shawi, R., Hafner, A., Olsen, J., Chun, S., Raza, S., Thrasivoulou, C., et al. (2008). Neurotoxic and neurotrophic roles of proNGF and the receptor sortilin in the adult and ageing nervous system. Eur. J. Neurosci. 27, 2103-2114. doi: 10.1111/j.1460-9568.2008.06152.x

Alzheimer, A. (1911). Uber eigenartige krankheitsfaelle des spaetern alters, zeitschrift fuer die gesamte. Neurol. Psych. 4, 256-286.

Alzheimer's Association (2019). 2018 Alzheimer's disease facts and figures. Alzheimer's Dement. 14, 367-429. doi: 10.1016/j.jalz.2018.02.001

Amadoro, G., Corsetti, V., Ciotti, M. T., Florenzano, F., Capsoni, S., Amato, G., et al. (2011). Endogenous abeta causes cell death via early tau hyperphosphorylation. Neurobiol. Aging 32, 969-990. doi: 10.1016/j. neurobiolaging.2009.06.005

Anastasia, A., Deinhardt, K., Chao, M. V., Will, N. E., Irmady, K., Lee, F. S., et al. (2013). Val66Met polymorphism of BDNF alters prodomain structure to induce neuronal growth cone retraction. Nat. Commun. 4:2490. doi: 10.1038/ ncomms 3490

Andersson, C., Hansson, O., Minthon, L., Andreasen, N., Blennow, K., Zetterberg, H., et al. (2016). A genetic variant of the sortilin 1 gene is associated with reduced risk of alzheimer's disease. J. Alzheimers Dis. 53, 1353-1363. doi: 10. 3233/JAD- 160319

Apfel, S. C. (2001). Neurotrophic factor therapy-prospects and problems. Clin. Chem. Lab. Med. 39, 351-355.

Arriagada, P., Growdon, J., Hedley-Whyte, E., and Hyman, B. (1992). Neurofibrillary tangles but not senile plaques parallel duration and severity of Alzheimer's disease. Neurology 42, 631-639.

Babu, J. R., Geetha, T., and Wooten, M. W. (2005). Sequestosome 1/p62 shuttles polyubiquitinated tau for proteasomal degradation. J. Neurochem. 94, 192-203. doi: 10.1111/j.1471-4159.2005.03181.x

Ballinger, E. C., Ananth, M., Talmage, D. A., and Role, L. W. (2016). Basal forebrain cholinergic circuits and signaling in cognition and cognitive decline. Neuron 91 , 1199-1218. doi: 10.1016/j.neuron.2016.09.006

Bamji, S. X., Majdan, M., Pozniak, C. D., Belliveau, D. J., Aloyz, R., Kohn, J., et al. (1998). The p75 neurotrophin receptor mediates neuronal apoptosis and is essential for naturally occurring sympathetic neuron death. J. Cell Biol. 140, 911-923. doi: 10.1083/jcb.140.4.911

Bartus, R. T., Dean, R. L. III, Beer, B., and Lippa, A. S. (1982). The cholinergic hypothesis of geriatric memory dysfunction. Science 217, 408-414. doi: 10. 1126/science.7046051

Bekdash, R. (2016). "Choline and the Brain: An Epigenetic Perspective," in The Benefits of Natural Products for Neurodegenerative Diseases, eds A. M. EssaM and G. Guillemin (Cham: Springer), 381-399. doi: 10.1007/978-3-319-28383$8 \_21$

Berger, Z., Roder, H., Hanna, A., Carlson, A., Rangachari, V., Yue, M., et al. (2007). Accumulation of pathological tau species and memory loss in a conditional model of tauopathy. J. Neurosci. 27, 3650-3662. doi: 10.1523/JNEUROSCI. 0587-07.2007

Bertrand, J., Plouffe, V., Senechal, P., and Leclerc, N. (2010). The pattern of human tau phosphorylation is the result of priming and feedback events in primary hippocampal neurons. Neuroscience 168, 323-334. doi: 10.1016/j.neuroscience. 2010.04.009

Blesch, A., and Tuszynski, M. (1995). Ex vivo gene therapy for Alzheimer's disease and spinal cord injury. Clin. Neurosci. 3, 268-274.

Blocq, P., and Marinesco, G. (1892). Sur les lésions et la pathogénie de l'épilepsie dite essentielle. Sem. Med. 12, 445-456.

Braak, H., and Braak, E. (1991). Neuropathological stageing of Alzheimer-related changes. Acta Neuropathol. 82, 239-259. doi: 10.1007/bf00308809
Bronfman, F. C., and Fainzilber, M. (2004). Multi-tasking by the p75 neurotrophin receptor: sortilin things out? EMBO Rep. 5, 867-871. doi: 10.1038/sj.embor. 7400219

Bruno, M. A., Clarke, P. B., Seltzer, A., Quirion, R., Burgess, K., Cuello, A. C., et al. (2004). Long-lasting rescue of age-associated deficits in cognition and the CNS cholinergic phenotype by a partial agonist peptidomimetic ligand of TrkA. J. Neurosci. 24, 8009-8018. doi: 10.1523/jneurosci.1508-04.2004

Bruno, M. A., Counts, S. E., Mufson, E. J., and Cuello, A. C. (2007). Increased MMP-9 cortical level and activity in subjects with Mild cognitive Impairment. Soc. Neurosci. 68, 1309-1318.

Bruno, M. A., and Cuello, A. C. (2006). Activity-dependent release of precursor nerve growth factor, conversion to mature nerve growth factor, and its degradation by a protease cascade. Proc. Natl. Acad. Sci. U.S.A. 103, 6735-6740. doi: 10.1073/pnas.0510645103

Bruno, M. A., Leon, W. C., Fragoso, G., Mushynski, W. E., Almazan, G., and Cuello, A. C. (2009). Amyloid beta-induced nerve growth factor dysmetabolism in Alzheimer disease. J. Neuropathol. Exp. Neurol. 68, 857-869. doi: 10.1097/ NEN.0b013e3181aed9e6

Burgos, I., Cuello, A. C., Liberini, P., Pioro, E., and Masliah, E. (1995). NGFmediated synaptic sprouting in the cerebral cortex of lesioned primate brain. Brain Res. 692, 154-160. doi: 10.1016/0006-8993(95)00696-n

Canu, N., Amadoro, G., Triaca, V., Latina, V., Sposato, V., Corsetti, V., et al. (2017). The intersection of NGF/TrkA signaling and amyloid precursor protein processing in alzheimer's disease neuropathology. Int. J. Mol. Sci. 18:1319. doi: 10.3390/ijms18061319

Capsoni, S., Ugolini, G., Comparini, A., Ruberti, F., Berardi, N., and Cattaneo, A. (2000). Alzheimer-like neurodegeneration in aged antinerve growth factor transgenic mice. Proc. Natl. Acad. Sci. U.S.A. 97, 6826-6831. doi: 10.1073/pnas. 97.12.6826

Casaccia-Bonnefil, P., Carter, B. D., Dobrowsky, R. T., and Chao, M. V. (1996). Death of oligodendrocytes mediated by the interaction of nerve growth factor with its receptor p75. Nature 383, 716-719. doi: 10.1038/383716a0

Cavedo, E., Grothe, M., Colliot, O., Lista, S., Chupin, M., Dormont, D., et al. (2017). Reduced basal forebrain atrophy progression in a randomized donepezil trial in prodromal Alzheimer's disease. Sci. Rep. 7, 1-10.

Chao, M. (2003). Neurotrophins and their receptors: a convergence point for many signalling pathways. Nat. Rev. Neurosci. 4, 299-309. doi: 10.1038/nrn1078

Charles, V., Mufson, E. J., Friden, P. M., Bartus, R. T., and Kordower, J. H. (1996). Atrophy of cholinergic basal forebrain neurons following excitotoxic cortical lesions is reversed by intravenous administration of an NGF conjugate. Brain Res. 728, 193-203. doi: 10.1016/s0006-8993(96)00398-8

Chen, S., Owens, G. C., Makarenkova, H., and Edelman, D. B. (2010). HDAC6 regulates mitochondrial transport in hippocampal neurons. PLoS One 5:e10848. doi: 10.1371/journal.pone.0010848

Christensen, D. D. (2007). Alzheimer's disease: progress in the development of anti-amyloid disease-modifying therapies. CNS Spectr. 12, 113-116, 119-123.

Cook, C., Gendron, T. F., Scheffel, K., Carlomagno, Y., Dunmore, J., DeTure, M., et al. (2012). Loss of HDAC6, a novel CHIP substrate, alleviates abnormal tau accumulation. Hum. Mol. Genet. 21, 2936-2945. doi: 10.1093/hmg/dds125

Coulson, E., and Nykjaer, A. (2013). Up-regulation of sortilin mediated by amyloid$\beta$ and p75NTR: safety lies in the middle course. J. Neurochem. 127, 149-151. doi: $10.1111 /$ jnc. 12389

Counts, S. E., Che, S., Ginsberg, S. D., and Mufson, E. J. (2011). Gender differences in neurotrophin and glutamate receptor expression in cholinergic nucleus basalis neurons during the progression of Alzheimer's disease. J. Chem. Neuroanat. 42, 111-117. doi: 10.1016/j.jchemneu.2011. 02.004

Counts, S. E., He, B., Prout, J. G., Michalski, B., Farotti, L., Fahnestock, M., et al. (2016). Cerebrospinal fluid proNGF: a putative biomarker for early Alzheimer's disease. Curr. Alzheimer Res. 13, 800-808. doi: 10.2174/ 1567205013666160129095649

Counts, S. E., Nadeem, M., Wuu, J., Ginsberg, S. D., Saragovi, H. U., and Mufson, E. J. (2004). Reduction of cortical TrkA but not p75(NTR) protein in early-stage Alzheimer's disease. Ann. Neurol. 56, 520-531. doi: 10.1002/ana.20233

Cozza, A., Melissari, E., Iacopetti, P., Mariotti, V., Tedde, A., Nacmias, B., et al. (2008). SNPs in neurotrophin system genes and Alzheimer's disease in an Italian population. J. Alzheimers Dis. 15, 61-70. doi: 10.3233/jad-200815105 
Crutcher, K. A., Scott, S. A., Liang, S., Everson, W. V., and Weingartner, J. (1993). Detection of NGF-like activity in human brain tissue: Increased levels in Alzheimer's disease. J. Neurosci. 13, 2540-2550. doi: 10.1523/jneurosci.1306-02540.1993

Dawson, M. A., and Kouzarides, T. (2012). Cancer epigenetics: from mechanism to therapy. Cell 150, 12-27. doi: 10.1016/j.cell.2012.06.013

DeKosky, S. T., Ikonomovic, M. D., Styren, S. D., Beckett, L., Wisniewski, S., Bennett, D. A., et al. (2002). Upregulation of choline acetyltransferase activity in hippocampus and frontal cortex of elderly subjects with mild cognitive impairment. Ann. Neurol. 51, 145-155. doi: 10.1002/ana. 10069

Di Maria, E., Giorgio, E., Uliana, V., Bonvicini, C., Faravelli, F., Cammarata, S., et al. (2012). Possible influence of a non-synonymous polymorphism located in the NGF precursor on susceptibility to late-onset Alzheimer's disease and mild cognitive impairment. J. Alzheimers Dis. 29, 699-705. doi: 10.3233/JAD-2012112006

Ding, H., Dolan, P. J., and Johnson, G. V. (2008). Histone deacetylase 6 interacts with the microtubule-associated protein tau. J. Neurochem. 106, 2119-2130. doi: 10.1111/j.1471-4159.2008.05564.x

Douchamps, V., and Mathis, C. (2017). A second wind for the cholinergic system in Alzheimer's therapy. Behav. Pharmacol. 28, 112-123. doi: 10.1097/FBP. 0000000000000300

Edwards, R. H., Selby, M. J., Garcia, P. D., and Rutter, W. J. (1988). Processing of the native nerve growth factor precursor to form biologically active nerve growth factor. J. Biol. Chem. 263, 6810-6815.

Fahnestock, M., Michalski, B., Xu, B., and Coughlin, M. (2001). The precursor pro-nerve growth factor is the predominant form of nerve growth factor in brain and is increased in Alzheimer's disease. Mol. Cell Neurosci. 18, 210-220. doi: 10.1006/mcne.2001.1016

Fahnestock, M., Scott, S. A., Jette, N., Weingartner, J. A., and Crutcher, K. A. (1996). Nerve growth factor mRNA and protein levels measured in the same tissue from normal and Alzheimer's disease parietal cortex. Brain Res. Mol. Brain Res. 42, 175-178. doi: 10.1016/s0169-328x(96)00193-3

Fahnestock, M., Yu, G., Michalski, B., Mathew, S., Colquhoun, A., Ross, G. M., et al. (2004). The nerve growth factor precursor proNGF exhibits neurotrophic activity but is less active than mature nerve growth factor. J. Neurochem. 89, 581-592. doi: 10.1111/j.1471-4159.2004.02360.x

Frade, J. M. (2000). Unscheduled re-entry into the cell cycle induced by NGF precedes cell death in nascent retinal neurones. J. Cell Sci. 113(Pt 7), 1139-1148.

Francke, U., Martinville, B. D., Coussens, L., and Ullrich, A. (1983). The human gene for the beta subunit of nerve growth factor is located on the proximal short arm of chromosome 1. Science 222, 1248-1251. doi: 10.1126/science.6648531

Friedman, W. J. (2000). Neurotrophins induce death of hippocampal neurons via the p75 receptor. J. Neurosci. 20, 6340-6346. doi: 10.1523/jneurosci.20-1706340.2000

Gage, F. H., Rosenberg, M. B., Tuszynski, M. H., Yoshida, K., Armstrong, D. M., Hayes, R. C., et al. (1990). Gene therapy in the CNS: intracerebral grafting of genetically modified cells. Prog. Brain Res. 86, 205-217. doi: 10.1016/s00796123(08)63178-7

Gage, F. H., Tuszynski, M. H., Chen, K. S., Armstrong, D., and Buzsaki, G. (1989). Survival, growth and function of damaged cholinergic neurons. EXS 57, 259-274. doi: 10.1007/978-3-0348-9138-7_26

Gilmor, M. L., Erickson, J. D., Varoqui, H., Hersh, L. B., Bennett, D. A., Cochran, E. J., et al. (1999). Preservation of nucleus basalis neurons containing choline acetyltransferase and the vesicular acetylcholine transporter in the elderly with mild cognitive impairment and early Alzheimer's disease. J. Comp. Neurol. 411, 693-704. doi: 10.1002/(sici)1096-9861(19990906)411\%3A4\% 3C693\%3A\%3Aaid-cne13\%3E3.3.co\%3B2-4

Ginsberg, S., Malek-Ahmadi, M., Alldred, M., Che, S., Elarova, I., Chen, Y., et al. (2019). Selective decline of neurotrophin and neurotrophin receptor genes within CA1 pyramidal neurons and hippocampus proper: correlation with cognitive performance and neuropathology in mild cognitive impairment and Alzheimer's disease. Hippocampus 29, 422-439. doi: 10.1002/hipo.22802

Ginsberg, S., and Martin, L. (1998). Ultrastructural analysis of the progression of neurodegeneration in the septum following fimbria-fornix transection. Neuroscience 86, 1259-1272. doi: 10.1016/s0306-4522(98)00136-5

Ginsberg, S. D., Alldred, M. J., Counts, S. E., Cataldo, A. M., Neve, R. L., Jiang, Y., et al. (2010). Microarray analysis of hippocampal CA1 neurons implicates early endosomal dysfunction during Alzheimer's disease progression. Biol. Psychiatry 68, 885-893. doi: 10.1016/j.biopsych.2010.05.030

Ginsberg, S. D., Che, S., Counts, S. E., and Mufson, E. J. (2006a). Shift in the ratio of three-repeat tau and four-repeat tau mRNAs in individual cholinergic basal forebrain neurons in mild cognitive impairment and Alzheimer's disease. J. Neurochem. 96, 1401-1408. doi: 10.1111/j.1471-4159.2005.03641.x

Ginsberg, S. D., Che, S., Wuu, J., Counts, S. E., and Mufson, E. J. (2006b). Down regulation of trk but not p75NTR gene expression in single cholinergic basal forebrain neurons mark the progression of Alzheimer's disease. J. Neurochem. 97, 475-487. doi: 10.1111/j.1471-4159.2006.03764.x

Ginsberg, S. D., Hemby, S. E., Lee, V. M., Eberwine, J. H., and Trojanowski, J. Q. (2000). Expression profile of transcripts in Alzheimer's disease tangle-bearing CA1 neurons. Ann. Neurol. 48, 77-87. doi: 10.1002/1531-8249(200007)48\% 3A1\%3C77\%3A\%3Aaid-ana12\%3E3.3.co\%3B2-1

Gnahn, H., Hefti, F., Heumann, R., Schwab, M. E., and Thoenen, H. (1983). NGF-mediated increase of choline acetyltransferase (ChAT) in the neonatal rat forebrain: evidence for a physiological role of NGF in the brain? Brain Res. 285, 45-52. doi: 10.1016/0165-3806(83)90107-4

Goedert, M., Fine, A., Dawbarn, D., Wilcock, G. K., and Chao, M. V. (1989). Nerve growth factor receptor mRNA distribution in human brain: normal levels in basal forebrain in Alzheimer's disease. Mol. Brain Res. 5, 1-7. doi: 10.1016/ 0169-328x(89)90011-9

Goedert, M., Hasegawa, M., Jakes, R., Lawler, S., Cuenda, A., and Cohen, P. (1997). Phosphorylation of microtubule-associated protein tau by stress-activated protein kinases. FEBS Lett. 409, 57-62. doi: 10.1016/s0014-5793(97)00483-3

Graff, J., Rei, D., Guan, J. S., Wang, W. Y., Seo, J., Hennig, K. M., et al. (2012). An epigenetic blockade of cognitive functions in the neurodegenerating brain. Nature 483, 222-226. doi: 10.1038/nature10849

Graff, J., and Tsai, L. H. (2013). Histone acetylation: molecular mnemonics on the chromatin. Nat. Rev. Neurosci. 14, 97-111. doi: 10.1038/nrn3427

Grothe, M., Heinsen, H., and Teipel, S. J. (2012). Atrophy of the cholinergic Basal forebrain over the adult age range and in early stages of Alzheimer's disease. Biol. Psychiatry 71, 805-813. doi: 10.1016/j.biopsych.2011.06.019

Guan, J. S., Haggarty, S. J., Giacometti, E., Dannenberg, J. H., Joseph, N., Gao, J., et al. (2009). HDAC2 negatively regulates memory formation and synaptic plasticity. Nature 459, 55-60. doi: 10.1038/nature07925

Guillozet, A. L., Weintraub, S., Mash, D. C., and Mesulam, M. M. (2003). Neurofibrillary tangles, amyloid, and memory in aging and mild cognitive impairment. Arch. Neurol. 60, 729-736. doi: 10.1001/archneur.6 0.5.729

Hampel, H., Mesulam, M. M., Cuello, A. C., Farlow, M. R., Giacobini, E., Grossberg, G. T., et al. (2018). The cholinergic system in the pathophysiology and treatment of Alzheimer's disease. Brain 141, 1917-1933. doi: 10.1093/brain/aw y132

Hampel, H., Schneider, L. S., Giacobini, E., Kivipelto, M., Sindi, S., Dubois, B., et al. (2015). Advances in the therapy of Alzheimer's disease: targeting amyloid beta and tau and perspectives for the future. Expert. Rev. Neurother. 15, 83-105. doi: $10.1586 / 14737175.2015 .995637$

Hardy, J., and Selkoe, D. J. (2002). The amyloid hypothesis of Alzheimer's disease: progress and problems on the road to therapeutics. Science 297, 353-356. doi: 10.1126/science. 1072994

Harrington, A., Kim, J. Y., and Yoon, S. (2002). Activation of Rac GTPase by p75 is necessary for c-jun N-terminal kinase-mediated apoptosis. J. Neurosci. 22, 156-166. doi: 10.1523/jneurosci.22-01-00156.2002

Hartikka, J., and Hefti, F. (1988). Comparison of nerve growth factor's effects on development of septum, striatum, and nucleus basalis cholinergic neurons in vitro. J. Neurosci. Res. 21, 352-364. doi: 10.1002/jnr.490210227

Hatanaka, H., Nihonmatsu, I., and Tsukui, H. (1988). Nerve growth factor promotes survival of cultured magnocellular cholinergic neurons from nucleus basalis of Meynert in postnatal rats. Neurosci. Lett. 90, 63-68. doi: 10.1016/ 0304-3940(88)90787-2

Hefti, F. (1986). Nerve growth factor promotes survival of septal cholinergic neurons after fimbrial transections. J. Neurosci. 6, 2155-2162. doi: 10.1523/ jneurosci.06-08-02155.1986

Hefti, F., Hartikka, J., and Knusel, B. (1989). Function of neurotrophic factors in the adult and aging brain and their possible use in the treatment of neurodegenerative diseases. Neurobiol. Aging 10, 515-533. doi: 10.1016/01974580(89)90118-8 
Hefti, F., and Mash, D. C. (1989). Localization of nerve growth factor receptors in the normal human brain and in Alzheimer's disease. Neurobiol. Aging 10, 75-87. doi: 10.1016/s0197-4580(89)80014-4

Hellweg, R., Gericke, C. A., Jendroska, K., Hartung, H. D., and Cervos-Navarro, J. (1998). NGF content in the cerebral cortex of non-demented patients with amyloid-plaques and in symptomatic Alzheimer's disease. Int. J. Dev. Neurosci. 16, 787-794. doi: 10.1016/s0736-5748(98)00088-4

Henry, J. M. (1998). Neurons and nobel prizes: a centennial history of neuropathology. Neurosurgery 42, 143-155; discussion155-146.

Higgins, G. A., Koh, S., Chen, K. S., and Gage, F. H. (1989). NGF induction of NGF receptor gene expression and cholinergic neuronal hypertrophy within the basal forebrain of the adult rat. Neuron 3, 247-256. doi: 10.1016/0896-6273(89) 90038- $\mathrm{x}$

Ho, A., Moore, R., Lopez, O., Kuller, L., and Becker, J. (2008). Basal forebrain atrophy is a presymptomatic marker for alzheimer's disease. Alzheimers Dement. 4, 271-279. doi: 10.1016/j.jalz.2008.04.005

Hock, C., Heese, K., Muller-Spahn, F., Huber, P., Riesen, W., Nitsch, R. M., et al. (2000). Increased CSF levels of nerve growth factor in patients with Alzheimer's disease. Neurology 54, 2009-2011. doi: 10.1212/wnl.54.10.2009

Humpel, C., and Weis, C. (2002). "Nerve growth factor and cholinergic CNS neurons studied in organotypic brain slices," in Ageing and Dementia Current and Future Concepts. Journal of Neural Transmission, Vol. 62, eds K. A. Jellinger, R. Schmidt, and M. Windisch (Vienna: Springer).

Hyman, B., Hoesen, G. V., Damasio, A., and Barnes, C. (1984). Alzheimer's disease: cell-specific pathology isolates the hippocampal formation. Science 225, 1168-1170. doi: 10.1126/science.6474172

Hyman, B. T., Van Hoesen, G. W., and Damasio, A. R. (1990). Memory-related neural systems in Alzheimer's disease: an anatomic study. Neurology 40, 1721-1730.

Ibanez, C. F. (2002). Jekyll-Hyde neurotrophins: the story of proNGF. Trends Neurosci. 25, 284-286. doi: 10.1016/s0166-2236(02)02169-0

Ibanez, C. F., and Simi, A. (2012). p75 neurotrophin receptor signaling in nervous system injury and degeneration: paradox and opportunity. Trends Neurosci. 35, 431-440. doi: 10.1016/j.tins.2012. 03.007

Iqbal, K., Gong, C. X., and Liu, F. (2013). Hyperphosphorylation-induced tau oligomers. Front. Neurol. 4:112. doi: 10.3389/fneur.2013.00112

Iulita, M. F., Do Carmo, S., Ower, A. K., Fortress, A. M., Flores Aguilar, L., Hanna, M., et al. (2014). Nerve growth factor metabolic dysfunction in down's syndrome brains. Brain 137(Pt 3), 860-872. doi: 10.1093/brain/aw t372

James, M. L., Belichenko, N. P., Shuhendler, A. J., Hoehne, A., Andrews, L. E., Condon, C., et al. (2017). [(18)F]GE-180 PET detects reduced microglia activation after LM11A-31 therapy in a mouse model of alzheimer's disease. Theranostics 7, 1422-1436. doi: 10.7150/thno.17666

Jang, S. W., Okada, M., Sayeed, I., Xiao, G., Stein, D., Jin, P., et al. (2007). Gambogic amide, a selective agonist for TrkA receptor that possesses robust neurotrophic activity, prevents neuronal cell death. Proc. Natl. Acad. Sci. U.S.A. 104, 16329-16334. doi: 10.1073/pnas.0706662104

Jette, N., Cole, M. S., and Fahnestock, M. (1994). NGF mRNA is not decreased in frontal cortex from Alzheimer's disease patients. Brain Res. Mol. Brain Res. 25, 242-250. doi: 10.1016/0169-328x(94)90159-7

Johannsen, P. (2006). Assessing therapeutic efficacy in a progressive disease: a study of donepezil in Alzheimer's disease. CNS Drugs 20, 311-325. doi: 10.2165/ 00023210-200620040-00005

Johnson, J., Pa, J., Boxer, A., Kramer, J., Freeman, K., and Yaffe, K. (2010). Baseline predictors of clinical progression among patients with dysexecutive mild cognitive impairment. Dement. Geriatr. Cogn. Disord. 30, 344-351. doi: $10.1159 / 000318836$

Jonhagen, M. E. (2000). Nerve growth factor treatment in dementia. Alzheimer Dis. Assoc. Disord. 14(Suppl. 1), S31-S38.

Julien, C., Tremblay, C., Emond, V., Lebbadi, M., Salem, N, Jr., Bennett, D. A., et al. (2009). SIRT1 decrease parallels the accumulation of tau in Alzheimer disease. J. Neuropathol. Exp. Neurol. 68, 48-58. doi: 10.1097/NEN.0b013e31819 22348

Kaplan, D. R., and Miller, F. D. (2004). Neurobiology: a move to sort life from death. Nature 427, 798-799. doi: 10.1038/427798a
Knowles, J., Rajadas, J., Nguyen, T., Yang, T., LeMieux, M., Griend, L. V., et al. (2009). The p75 neurotrophin receptor promotes amyloid- $\beta(1-42)-$ induced neuritic dystrophy in vitro and in vivo. J. Neurosci. 29, 10627-10637. doi: 10.1523/JNEUROSCI.0620-09.2009

Knowles, J. K., Simmons, D. A., Nguyen, T. V., Vander Griend, L., Xie, Y., Zhang, H., et al. (2013). Small molecule p75NTR ligand prevents cognitive deficits and neurite degeneration in an Alzheimer's mouse model. Neurobiol. Aging 34, 2052-2063. doi: 10.1016/j.neurobiolaging.2013.02.015

Kopeikina, K. J., Carlson, G. A., Pitstick, R., Ludvigson, A. E., Peters, A., Luebke, J. I., et al. (2011). Tau accumulation causes mitochondrial distribution deficits in neurons in a mouse model of tauopathy and in human Alzheimer's disease brain. Am. J. Pathol. 179, 2071-2082. doi: 10.1016/j.ajpath.2011.07.004

Lalla, R., and Donmez, G. (2013). The role of sirtuins in Alzheimer's disease. Front. Aging Neurosci. 5:16. doi: 10.3389/fnagi.2013.00016

Lasagna-Reeves, C. A., Castillo-Carranza, D. L., Sengupta, U., Guerrero-Munoz, M. J., Kiritoshi, T., Neugebauer, V., et al. (2012). Alzheimer brain-derived tau oligomers propagate pathology from endogenous tau. Sci. Rep. 2:700. doi: 10.1038/srep00700

Lee, R., Kermani, P., Teng, K. K., and Hempstead, B. L. (2001). Regulation of cell survival by secreted proneurotrophins. Science 294, 1945-1948. doi: 10.1126/ science. 1065057

Levi-Montalcini, R. (2000). From Turin to Stockholm via St. Louis and Rio de Janeiro. Science 287:809. doi: 10.1126/science.287.5454.809

Longo, F. M., Yang, T., Knowles, J. K., Xie, Y., Moore, L. A., and Massa, S. M. (2007). Small molecule neurotrophin receptor ligands: novel strategies for targeting Alzheimer's disease mechanisms. Curr. Alzheimer Res. 4, 503-506. doi: 10.2174/156720507783018316

Lorenzl, S., Albers, D. S., LeWitt, P. A., Chirichigno, J. W., Hilgenberg, S. L., Cudkowicz, M. E., et al. (2003). Tissue inhibitors of matrix metalloproteinases are elevated in cerebrospinal fluid of neurodegenerative diseases. J. Neurol. Sci. 207, 71-76. doi: 10.1016/s0022-510x(02)00398-2

Lorenzl, S., Buerger, K., Hampel, H., and Beal, M. F. (2008). Profiles of matrix metalloproteinases and their inhibitors in plasma of patients with dementia. Int. Psychogeriatr. 20, 67-76. doi: 10.1017/s1041610207005790

Luna-Munoz, J., Chavez-Macias, L., Garcia-Sierra, F., and Mena, R. (2007). Earliest stages of tau conformational changes are related to the appearance of a sequence of specific phospho-dependent tau epitopes in Alzheimer's disease. J. Alzheimers Dis. 12, 365-375. doi: 10.3233/jad-2007-12410

Maeda, S., Sahara, N., Saito, Y., Murayama, M., Yoshiike, Y., Kim, H., et al. (2007). Granular tau oligomers as intermediates of tau filaments. Biochemistry 46, 3856-3861. doi: 10.1021/bi0613590

Mahady, L., Nadeem, M., Malek-Ahmadi, M., Chen, K., Perez, S., and Mufson, E. (2018). Frontal cortex epigenetic dysregulation during the progression of alzheimer's disease. J. Alzheimer's Dis. 62, 115-131. doi: 10.3233/JAD-171032

Mahady, L., Nadeem, M., Malek-Ahmadi, M., Chen, K., Perez, S. E., and Mufson, E. J. (2019). HDAC2 dysregulation in the nucleus basalis of meynert during the progression of Alzheimer's disease. Neuropathol. Appl. Neurobiol. 45, 380-397. doi: $10.1111 /$ nan. 12518

Mamidipudi, V., and Wooten, M. W. (2002). Dual role for p75(NTR) signaling in survival and cell death: can intracellular mediators provide an explanation? J. Neurosci. Res. 68, 373-384. doi: 10.1002/jnr.10244

Mangialasche, F., Solomon, A., Winblad, B., Mecocci, P., and Kivipelto, M. (2010). Alzheimer's disease: clinical trials and drug development. Lancet Neurol. 9, 702-716. doi: 10.1016/S1474-4422(10)70119-8

Mann, D. M., and Esiri, M. M. (1989). The pattern of acquisition of plaques and tangles in the brains of patients under 50 years of age with down's syndrome. J. Neurol. Sci. 89, 169-179. doi: 10.1016/0022-510x(89)90019-1

Markesbery, W. R. (2010). Neuropathologic alterations in mild cognitive impairment: a review. J. Alzheimers Dis. 19, 221-228. doi: 10.3233/JAD-20101220

Markesbery, W. R., Schmitt, F. A., Kryscio, R. J., Davis, D. G., Smith, C. D., and Wekstein, D. R. (2006). Neuropathologic substrate of mild cognitive impairment. Arch. Neurol. 63, 38-46. doi: 10.1001/archneur.63.1.38

Massa, S. M., Xie, Y., Yang, T., Harrington, A. W., Kim, M. L., Yoon, S. O., et al. (2006). Small, nonpeptide p75NTR ligands induce survival signaling and inhibit proNGF-induced death. J. Neurosci. 26, 5288-5300. doi: 10.1523/JNEUROSCI. 3547-05.2006 
Mastroeni, D., Grover, A., Delvaux, E., Whiteside, C., Coleman, P. D., and Rogers, J. (2010). Epigenetic changes in Alzheimer's disease: decrements in DNA methylation. Neurobiol. Aging 31, 2025-2037. doi: 10.1016/j.neurobiolaging. 2008.12.005

Matyi, J., Tschanz, J. T., Rattinger, G. B., Sanders, C., Vernon, E. K., Corcoran, C., et al. (2017). Sex differences in risk for alzheimer's disease related to neurotrophin gene polymorphisms: the cache county memory study. J. Gerontol. A Biol. Sci. Med. Sci. 72, 1607-1613. doi: 10.1093/gerona/glx092

McArthur, J. C., Yiannoutsos, C., Simpson, D. M., Adornato, B. T., Singer, E. J., Hollander, H., et al. (2000). A phase II trial of nerve growth factor for sensory neuropathy associated with HIV infection. AIDS Clinical Trials Group Team 291. Neurology 54, 1080-1088. doi: 10.1212/wnl.54.5.1080

McKhann, G., Drachman, D., Folstein, M., Katzman, R., Price, D., and Stadlan, E. M. (1984). Clinical diagnosis of Alzheimer's disease: report of the NINCDSADRDA Work Group under the auspices of department of health and human services task force on alzheimer's disease. Neurology 34, 939-944.

Mesulam, M., Shaw, P., Mash, D., and Weintraub, S. (2004). Cholinergic nucleus basalis tauopathy emerges early in the agingMCI-AD continuum. Ann. Neurol. 55, 815-828. doi: 10.1002/ana. 20100

Mesulam, M. M., Mufson, E. J., Levey, A. I., and Wainer, B. H. (1983). Cholinergic innervation of cortex by the basal forebrain: cytochemistry and cortical connections of the septal area, diagonal band nuclei, nucleus basalis (substantia innominata), and hypothalamus in the rhesus monkey. J. Comp. Neurol. 214, 170-197. doi: 10.1002/cne.902140206

Minnone, G., Soligo, M., Caiello, I., Prencipe, G., Manni, L., Marafon, D. P., et al. (2017). ProNGF-p75NTR axis plays a proinflammatory role in inflamed joints: a novel pathogenic mechanism in chronic arthritis. RMD Open 3:e000441. doi: 10.1136/rmdopen-2017-000441

Mufson, E. J., Binder, L., Counts, S. E., DeKosky, S. T., de Toledo-Morrell, L., Ginsberg, S. D., et al. (2012a). Mild cognitive impairment: pathology and mechanisms. Acta Neuropathol. 123, 13-30. doi: 10.1007/s00401-011-0884-1

Mufson, E. J., He, B., Nadeem, M., Perez, S., Counts, S., Leurgans, S., et al. (2012b). Hippocampal ProNGF signaling pathways and $\beta$-amyloid levels in mild cognitive impairment and alzheimer disease. J. Neuropathol. Exp. Neurol. 71, 1018-1029. doi: 10.1097/NEN.0b013e318272caab

Mufson, E. J., Malek-Ahmadi, M., Perez, S., and Chen, K. (2016a). Braak staging, plaque pathology and APOE status in elderly persons without cognitive impairment. Neurobiol. Aging 37, 147-153. doi: 10.1016/j.neurobiolaging.2015. 10.012

Mufson, E. J., Malek-Ahmadi, M., Snyder, N., Ausdemore, J., Chen, K., and Perez, S. E. (2016b). Braak stage and trajectory of cognitive decline in noncognitively impaired elders. Neurobiol. Aging 43, 101-110. doi: 10.1016/j.neurobiolaging. 2016.03.003

Mufson, E. J., Bothwell, M., and Kordower, J. H. (1989). Loss of nerve growth factor receptor-containing neurons in Alzheimer's disease: a quantitative analysis across subregions of the basal forebrain. Exp. Neurol. 105, 221-232. doi: 10. 1016/0014-4886(89)90124-6

Mufson, E. J., Counts, S. E., and Ginsberg, S. D. (2002a). Gene expression profiles of cholinergic nucleus basalis neurons in Alzheimer's disease. Neurochem. Res. 27, 1035-1048.

Mufson, E. J., Ma, S. Y., Dills, J., Cochran, E. J., Leurgans, S., Wuu, J., et al. (2002b). Loss of basal forebrain P75(NTR) immunoreactivity in subjects with mild cognitive impairment and Alzheimer's disease. J. Comp. Neurol. 443, 136-153. doi: $10.1002 /$ cne. 10122

Mufson, E. J., Ginsberg, S. D., Ikonomovic, M. D., and DeKosky, S. T. (2003). Human cholinergic basal forebrain: chemoanatomy and neurologic dysfunction. J. Chem. Neuroanat. 26, 233-242. doi: 10.1016/s0891-0618(03) 00068- 1

Mufson, E. J., and Kordower, J. H. (1992). Cortical neurons express nerve growth factor receptors in advanced age and Alzheimer disease. Proc. Natl. Acad. Sci. U.S.A. 89, 569-573. doi: 10.1073/pnas.89.2.569

Mufson, E. J., and Kordower, J. H. (1999). "Nerve growth factor in Alzheimer's disease," in Cerebral Cortex, eds A. A. Peter and J. H. Morrison (New York, NY: Kluwer Academic/Plenum Press), 681-731.

Mufson, E. J., Ma, S. J., Cochran, E. J., Bennett, D. A., Beckett, L. A., Jaffar, S., et al. (2000). Loss of nucleus basalis neurons containing trkA immunoreactivity in individuals with mild cognitive impairment and early Alzheimer's disease.
J. Comp. Neurol. 427, 19-30. doi: 10.1002/1096-9861(20001106)427\\%3A1\\% $3 \mathrm{C} 19 \backslash \% 3 \mathrm{~A} \backslash \% 3$ Aaid-cne $\backslash \% 3 \mathrm{E} 3.0$. co $\backslash \% 3 \mathrm{~B} 2$ - $\mathrm{a}$

Mufson, E. J., Mahady, L., Waters, D., Counts, S. E., Perez, S. E., DeKosky, S. T., et al. (2015). Hippocampal plasticity during the progression of Alzheimer's disease. Neuroscience 309, 51-67. doi: 10.1016/j.neuroscience.2015.03.006

Mufson, E. J., Wuu, J., Counts, S. E., and Nykjaer, A. (2010). Preservation of cortical sortilin protein levels in MCI and Alzheimer's disease. Neurosci. Lett. 47, 1129-1133. doi: 10.1016/j.neulet.2010.01.023

Murase, K., Nabeshima, T., Robitaille, Y., Quirion, R., Ogawa, M., and Hayashi, K. (1993). NGF level of is not decreased in the serum, brain-spinal fluid, hippocampus, or parietal cortex of individuals with Alzheimer's disease. Biochem. Biophys. Res. Commun. 193, 198-203. doi: 10.1006/bbrc.1993. 1609

Murphy, M., Wilson, Y. M., Vargas, E., Munro, K. M., Smith, B., Huang, A., et al. (2015). Reduction of p75 neurotrophin receptor ameliorates the cognitive deficits in a model of Alzheimer's disease. Neurobiol. Aging 36, 740-752. doi: 10.1016/j.neurobiolaging.2014.09.014

Murray, S. S., Perez, P., Lee, R., Hempstead, B. L., and Chao, M. V. (2004). A novel p75 neurotrophin receptor-related protein, $\mathrm{NRH} 2$, regulates nerve growth factor binding to the TrkA receptor. J. Neurosci. 24, 2742-2749. doi: 10.1523/JNEUROSCI.3960-03.2004

Nabeshima, T., Nitta, A., Fuji, K., Kameyama, T., and Hasegawa, T. (1994). Oral administration of NGF synthesis stimulators recovers reduced brain NGF content in aged rats and cognitive dysfunction in basal-forebrain-lesioned rats. Gerontology 40(Suppl. 2), 46-56. doi: 10.1159/000213627

Nair, J., Klaassen, A. L., Arato, J., Vyssotski, A. L., Harvey, M., and Rainer, G. (2018). Basal forebrain contributes to default mode network regulation. Proc. Natl. Acad. Sci. U.S.A. 115, 1352-1357. doi: 10.1073/pnas.1712431115

Narisawa-Saito, M., Wakabayashi, K., Tsuji, S., Takahashi, H., and Nawa, H. (1996). Regional specificity of alterations in NGF, BDNF and NT-3 levels in Alzheimer's disease. Neuroreport 7, 2925-2928. doi: 10.1097/00001756-19961125000024

Nguyen, T., Shen, L., Griend, L. V., Quach, L., Belichenko, N., Saw, N., et al. (2014). Small molecule p75NTR ligands reduce pathological phosphorylation and misfolding of tau, inflammatory changes, cholinergic degeneration, and cognitive deficits in $\mathrm{A} \beta \mathrm{PP}(\mathrm{L} / \mathrm{S})$ transgenic mice. J. Alzheimers Dis. 42, 459-483. doi: $10.3233 /$ jad- 140036

Nuydens, R., Dispersyn, G., de Jong, M., van den Kieboom, G., Borgers, M., and Geerts, H. (1997). Aberrant tau phosphorylation and neurite retraction during NGF deprivation in PC12 cells. Biochem. Biophys. Res. Commun. 240, 687-691. doi: 10.1006/bbrc.1997.7721

Nyborg, A. C., Ladd, T. B., Zwizinski, C. W., Lah, J. J., and Golde, T. E. (2006). Sortilin, SorCS1b, and SorLA Vps10p sorting receptors, are novel gammasecretase substrates. Mol. Neurodegener. 1:3.

Nykjaer, A., Lee, R., Teng, K. K., Jansen, P., Madsen, P., Nielsen, M. S., et al. (2004). Sortilin is essential for proNGF-induced neuronal cell death. Nature 427, 843-848. doi: 10.1038/nature02319

Nykjaer, A., Willnow, T., and Petersen, C. (2005). p75NTR-live or let die. Curr. Opin. Neurobiol. 15, 49-57. doi: 10.1016/j.conb.2005.01.004

Pandey, U., Nie, Z., Batlevi, Y., Mccray, B., Ritson, G., Nedelsky, N., et al. (2007). HDAC6 rescues neurodegeneration and provides an essential link between autophagy and the UPS. Nature 447, 859-863.

Patterson, K. R., Remmers, C., Fu, Y., Brooker, S., Kanaan, N. M., Vana, L., et al. (2011). Characterization of prefibrillar Tau oligomers in vitro and in Alzheimer disease. J. Biol. Chem. 286, 23063-23076. doi: 10.1074/jbc.M111.237974

Pedraza, C. E., Podlesniy, P., Vidal, N., Arevalo, J. C., Lee, R., Hempstead, B., et al. (2005). Pro-NGF isolated from the human brain affected by Alzheimer's disease induces neuronal apoptosis mediated by p75NTR. Am. J. Pathol. 166, 533-543. doi: 10.1016/S0002-9440(10)62275-4

Peng, S., Wuu, J., Mufson, E. J., and Fahnestock, M. (2004). Increased proNGF levels in subjects with mild cognitive impairment and mild alzheimer's disease. J. Neuropathol. Exp. Neurol. 63, 641-649. doi: 10.1093/jnen/63.6.641

Perez, S. E., He, B., Nadeem, M., Wuu, J., Scheff, S. W., Abrahamson, E. E., et al. (2015). Resilience of precuneus neurotrophic signaling pathways despite amyloid pathology in prodromal Alzheimer's disease. Biol. Psychiatry 77, 693-703. doi: 10.1016/j.biopsych.2013.12.016

Petersen, R. (2004). Mild cognitive impairment as a diagnostic entity. J. Intern. Med. 256, 183-194. doi: 10.1111/j.1365-2796.2004.01388.x 
Petersen, R. C., Smith, G. E., Waring, S. C., Ivnik, R. J., Tangalos, E. G., and Kokmen, E. (1999). Mild cognitive impairment: clinical characterization and outcome. Arch. Neurol. 56, 303-308.

Podlesniy, P., Kichev, A., Pedraza, C., Saurat, J., Encinas, M., Perez, B., et al. (2006). Pro-NGF from Alzheimer's disease and normal human brain displays distinctive abilities to induce processing and nuclear translocation of intracellular domain of p75NTR and apoptosis. Am. J. Pathol. 169, 119-131. doi: 10.2353/ajpath.2006.050787

Poirier, J., Delisle, M.-C., Quirion, R., Aubert, I., Farlow, M., Lahiri, D., et al. (1995). Apolipoprotein E4 allele as a predictor of cholinergic deficits and treatment outcome in alzheimer's disease. Proc. Natl. Acad. Sci. U.S.A. 92, 12260-12264. doi: $10.1073 /$ pnas.92.26.12260

Price, J. L., and Morris, J. C. (1999). Tangles and plaques in nondemented aging and "preclinical" Alzheimer's disease. Ann. Neurol. 45, 358-368. doi: 10.1002/ 1531-8249(199903)45\%3A3\%3C358\%3A\%3Aaid-ana12\%3E3.0.co\%3B2-x

Reitz, C., Tosto, G., Vardarajan, B., Rogaeva, E., Ghani, M., Rogers, R. S., et al. (2013). Independent and epistatic effects of variants in VPS10-d receptors on Alzheimer disease risk and processing of the amyloid precursor protein (APP). Transl. Psychiatry 3:e256. doi: 10.1038/tp.2013.13

Reynolds, C. H., Nebreda, A. R., Gibb, G. M., Utton, M. A., and Anderton, B. H. (1997). Reactivating kinase/p38 phosphorylates tau protein in vitro. J. Neurochem. 69, 191-198. doi: 10.1046/j.1471-4159.1997.69010191.x

Rosa, E., Mahendram, S., Ke, Y. D., Ittner, L. M., Ginsberg, S. D., and Fahnestock, M. (2016). Tau downregulates BDNF expression in animal and cellular models of Alzheimer's disease. Neurobiol. Aging 48, 135-142. doi: 10.1016/j. neurobiolaging.2016.08.020

Roux, P. P., and Barker, P. A. (2002). Neurotrophin signaling through the p75 neurotrophin receptor. Prog. Neurobiol. 67, 203-233. doi: 10.1016/s03010082(02)00016-3

Ryu, J. C., Tooke, K., Malley, S. E., Soulas, A., Weiss, T., Ganesh, N., et al. (2018). Role of proNGF/p75 signaling in bladder dysfunction after spinal cord injury. J. Clin. Invest. 128, 1772-1786. doi: 10.1172/JCI97837

Sadot, E., Heicklen-Klein, A., Barg, J., Lazarovici, P., and Ginzburg, I. (1996). Identification of a tau promoter region mediating tissue-specific-regulated expression in PC12 cells. J. Mol. Biol. 256, 805-812. doi: 10.1006/jmbi.1996. 0126

Sahara, N., DeTure, M., Ren, Y., Ebrahim, A. S., Kang, D., Knight, J., et al. (2013). Characteristics of TBS-extractable hyperphosphorylated tau species: aggregation intermediates in rTg4510 mouse brain. J. Alzheimers Dis. 33, 249-263. doi: 10.3233/JAD-2012-121093

Sassin, I., Schultz, C., Thal, D. R., Rub, U., Arai, K., Braak, E., et al. (2000). Evolution of Alzheimer's disease-related cytoskeletal changes in the basal nucleus of Meynert. Acta Neuropathol. 100, 259-269. doi: 10.1007/s004019900178

Schindowski, K., Belarbi, K., and Buee, L. (2008). Neurotrophic factors in Alzheimer's disease: role of axonal transport. Genes Brain Behav. 7(Suppl. 1), 43-56. doi: 10.1111/j.1601-183X.2007.00378.x

Schmitz, T. W., and Nathan Spreng, R. (2016). Basal forebrain degeneration precedes and predicts the cortical spread of Alzheimer's pathology. Nat. Commun. 7:13249. doi: 10.1038/ncomms13249

Schwab, M. E., Otten, U., Agid, Y., and Thoenen, H. (1979). Nerve growth factor (NGF) in the rat CNS: absence of specific retrograde axonal transport and tyrosine hydroxylase induction in locus coeruleus and substantia nigra. Brain Res. 168, 473-483. doi: 10.1016/0006-8993(79) 90303-2

Scott, S. A., Mufson, E., Weingartner, J., Skau, K., and Crutcher, K. (1995). Nerve growth factor in alzheimer's disease: increased levels throughout the brain coupled with declines in nucleus basalis. J. Neurosci. 15, 6213-6221. doi: 10 . 1523/jneurosci.15-09-06213.1995

Sendera, T., Ma, S., Jaffar, S., Kozlowski, P., Kordower, J., Mawal, Y., et al. (2000). Reduction in TrkA-immunoreactive neurons is not associated with an overexpression of galaninergic fibers within the nucleus basalis in down's syndrome. J. Neurochem. 74, 1185-1196. doi: 10.1046/j.1471-4159.2000. 741185.x

Shelton, S. B., and Johnson, G. V. (2001). Tau and HMW tau phosphorylation and compartmentalization in apoptotic neuronal PC12 cells. J. Neurosci. Res. 66, 203-213. doi: 10.1002/jnr.1212

Shen, L. L., Manucat-Tan, N. B., Gao, S. H., Li, W. W., Zeng, F., Zhu, C., et al. (2018). The ProNGF/p75NTR pathway induces tau pathology and is a therapeutic target for FTLD-tau. Mol. Psychiatry 23, 1813-1824. doi: 10.1038/ s41380-018-0071-z

Shen, X., Chen, J., Li, J., Kofler, J., and Herrup, K. (2016). Neurons in vulnerable regions of the alzheimer's disease brain display reduced ATM signaling. eNeuro 3:ENEURO.0124-15.2016. doi: 10.1523/ENEURO.0124-15.2016

Shoji, M., Golde, T. E., Ghiso, J., Cheung, T. T., Estus, S., Shaffer, L. M., et al. (1992). Production of the alzheimer amyloid beta protein by normal proteolytic processing. Science 258, 126-129. doi: 10.1126/science. 1439760

Simmons, D. A., Knowles, J. K., Belichenko, N. P., Banerjee, G., Finkle, C., Massa, S. M., et al. (2014). A small molecule p75NTR ligand, LM11A-31, reverses cholinergic neurite dystrophy in Alzheimer's disease mouse models with mid- to late-stage disease progression. PLoS One 9:e102136. doi: 10.1371/journal.pone. 0102136

Skaper, S. D. (2008). The biology of neurotrophins, signalling pathways, and functional peptide mimetics of neurotrophins and their receptors. CNS Neurol. Disord. Drug Targets 7, 46-62. doi: 10.2174/187152708783885174

Smith, D. E., Roberts, J., Gage, F. H., and Tuszynski, M. H. (1999). Age-associated neuronal atrophy occurs in the primate brain and is reversible by growth factor gene therapy. Proc. Natl. Acad. Sci. U.S.A. 96, 10893-10898. doi: 10.1073/pnas. 96.19.10893

Sotthibundhu, A., Sykes, A., Fox, B., Underwood, C., Thangnipon, W., and Coulson, E. (2008). Beta-amyloid(1-42) induces neuronal death through the p75 neurotrophin receptor. J. Neurosci. 28, 3941-3946. doi: 10.1523/ JNEUROSCI.0350-08.2008

Sperling, R., Mormino, E., and Johnson, K. (2014). The evolution of preclinical Alzheimer's disease: implications for prevention trials. Neuron 84, 608-622. doi: 10.1016/j.neuron.2014.10.038

Sramek, J. J., and Cutler, N. R. (1999). Recent developments in the drug treatment of Alzheimer's disease. Drugs Aging 14, 359-373. doi: 10.2165/00002512199914050-00004

Summers, W. K., Majovski, L. V., Marsh, G. M., Tachiki, K., and Kling, A. (1986). Oral tetrahydroaminoacridine in long-term treatment of senile dementia, Alzheimer type. N. Engl. J. Med. 315, 1241-1245. doi: 10.1056/ NEJM198611133152001

Teng, H. K., Teng, K. K., Lee, R., Wright, S., Tevar, S., Almeida, R. D., et al. (2005). ProBDNF induces neuronal apoptosis via activation of a receptor complex of p75NTR and sortilin. J. Neurosci. 25, 5455-5463. doi: 10.1523/jneurosci.512304.2005

Teng, K. K., and Hempstead, B. L. (2004). Neurotrophins and their receptors: signaling trios in complex biological systems. Cell Mol. Life Sci. 61, 35-48. doi: 10.1007/s00018-003-3099-3

Tep, C., Lim, T. H., Ko, P. O., Getahun, S., Ryu, J. C., Goettl, V. M., et al. (2013). Oral administration of a small molecule targeted to block proNGF binding to p75 promotes myelin sparing and functional recovery after spinal cord injury. J. Neurosci. 33, 397-410. doi: 10.1523/JNEUROSCI.0399-12. 2013

Thinakaran, G., and Koo, E. H. (2008). Amyloid precursor protein trafficking, processing, and function. J. Biol. Chem. 283, 29615-29619. doi: 10.1074/jbc. R800019200

Tiernan, C. T., Ginsberg, S. D., Guillozet-Bongaarts, A. L., Ward, S. M., He, B., Kanaan, N. M., et al. (2016). Protein homeostasis gene dysregulation in pretangle bearing nucleus basalis neurons during the progression of Alzheimer's disease. Neurobiol. Aging 42, 80-90. doi: 10.1016/j.neurobiolaging.2016. 02.031

Tiernan, C. T., Ginsberg, S. D., He, B., Ward, S. M., Guillozet-Bongaarts, A. L., Kanaan, N. M., et al. (2018a). Pretangle pathology within cholinergic nucleus basalis neurons coincides with neurotrophic and neurotransmitter receptor gene dysregulation during the progression of Alzheimer's disease. Neurobiol. Dis. 117, 125-136. doi: 10.1016/j.nbd.2018.05.021

Tiernan, C. T., Mufson, E. J., Kanaan, N. M., and Counts, S. E. (2018b). Tau oligomer pathology in nucleus basalis neurons during the progression of Alzheimer's disease. J. Neuropathol. Exp. Neurol. 77, 246-359. doi: 10.1016/j. nbd.2018.05.021

Trojanowski, J. Q., Schmidt, M. L., Shin, R. W., Bramblett, G. T., Rao, D., and Lee, V. M. (1993). Altered tau and neurofilament proteins in neurodegenerative diseases: diagnostic implications for Alzheimer's disease and Lewy body dementias. Brain Pathol. 3, 45-54. doi: 10.1111/j.1750-3639.1993. tb00725.x 
Troy, C. M., Friedman, J. E., and Friedman, W. J. (2002). Mechanisms of p75mediated death of hippocampal neurons. Role of caspases. J. Biol. Chem. 277, 34295-34302. doi: 10.1074/jbc.M205167200

Tuszynski, M., and Blesch, A. (2004). Nerve growth factor: from animal models of cholinergic neuronal degeneration to gene therapy in Alzheimer's disease. Prog. Brain Res. 146, 441-449.

Tuszynski, M. H., Hs, U., Amaral, D. G., and Gage, F. H. (1990). Nerve growth factor infusion in the primate brain reduces lesion-induced cholinergic neuronal degeneration. J. Neurosci. 10, 3604-3614. doi: 10.1523/jneurosci.1011-03604.1990

Tuszynski, M. H., Roberts, J., Senut, M. C., Hs, U., and Gage, F. H. (1996). Gene therapy in the adult primate brain: intraparenchymal grafts of cells genetically modified to produce nerve growth factor prevent cholinergic neuronal degeneration. Gene Ther. 3, 305-314.

Tuszynski, M. H., Sang, H., Yoshida, K., and Gage, F. H. (1991). Recombinant human nerve growth factor infusions prevent cholinergic neuronal degeneration in the adult primate brain. Ann. Neurol. 30, 625-636. doi: 10.1002/ana.410300502

Tuszynski, M. H., Thal, L., Pay, M., Salmon, D. P., Hs, U., Bakay, R., et al. (2005). A phase 1 clinical trial of nerve growth factor gene therapy for Alzheimer disease. Nat. Med. 11, 551-555. doi: 10.1038/nm1239

Tuszynski, M. H., Yang, J. H., Barba, D., Hs, U., Bakay, R. A., Pay, M. M., et al. (2015). Nerve growth factor gene therapy: activation of neuronal responses in alzheimer disease. JAMA Neurol. 72, 1139-1147. doi: 10.1001/jamaneurol.2015. 1807

Ulrich, E., Duwel, A., Kauffmann-Zeh, A., Gilbert, C., Lyon, D., Rudkin, B., et al. (1998). Specific TrkA survival signals interfere with different apoptotic pathways. Oncogene 16, 825-832. doi: 10.1038/sj.onc. 1201842

Vana, L., Kanaan, N. M., Ugwu, I. C., Wuu, J., Mufson, E. J., and Binder, L. I. (2011). Progression of tau pathology in cholinergic Basal forebrain neurons in mild cognitive impairment and Alzheimer's disease. Am. J. Pathol. 179, 2533-2550. doi: 10.1016/j.ajpath.2011.07.044

Volmar, C. H., and Claes, W. (2015). Histone deacetylases (HDACs) and brain function. Neuroepigenetics 1, 20-27. doi: 10.1016/j.nepig.2014. 10.002

Ward, S. M., Himmelstein, D. S., Lancia, J. K., Fu, Y., Patterson, K. R., and Binder, L. I. (2013). TOC1: characterization of a selective oligomeric tau antibody. J. Alzheimers Dis. 37, 593-602. doi: 10.3233/JAD-131235

Williams, L. R., Varon, S., Peterson, G. M., Wictorin, K., Fischer, W., Bjorklund, A., et al. (1986). Continuous infusion of nerve growth factor prevents basal forebrain neuronal death after fimbria fornix transection. Proc. Natl. Acad. Sci. U.S.A. 83, 9231-9235. doi: 10.1073/pnas.83.23.9231

Wimo, A. (2007). Clinical and economic outcomes-friend or foe? Int. Psychogeriatr. 19, 497-507. doi: 10.1017/s1041610207004930

Wimo, A., Guerchet, M., Ali, G. C., Wu, Y. T., Prina, A. M., Winblad, B., et al. (2017). The worldwide costs of dementia 2015 and comparisons with 2010. Alzheimers Dement. 13, 1-7. doi: 10.1016/j.jalz.2016. 07.150
Wu, C. K., Thal, L., Pizzo, D., Hansen, L., Masliah, E., and Geula, C. (2005). Apoptotic signals within the basal forebrain cholinergic neurons in Alzheimer's disease. Exp. Neurol. 195, 484-496. doi: 10.1016/j.expneurol.2005.06.020

Xu, K., Dai, X. L., Huang, H. C., and Jiang, Z. F. (2011). Targeting HDACs: a promising therapy for Alzheimer's disease. Oxid. Med. Cell Longev. 2011:143269. doi: 10.1155/2011/143269

Yang, T., Knowles, J., Lu, Q., Zhang, H., Arancio, O., Moore, L., et al. (2008). Small molecule, non-peptide p75NTR ligands inhibit a $\beta$-induced neurodegeneration and synaptic impairment. PLoS One 3: e3604. doi: 10.1371/journal.pone. 0003604

Yoon, S. O., Casaccia-Bonnefil, P., Carter, B., and Chao, M. V. (1998). Competitive signaling between TrkA and p75 nerve growth factor receptors determines cell survival. J. Neurosci. 18, 3273-3281. doi: 10.1523/jneurosci.18-09-03273.1998

Yoshiyama, Y., Lee, V. M., and Trojanowski, J. Q. (2013). Therapeutic strategies for tau mediated neurodegeneration. J. Neurol. Neurosurg. Psychiatry 84, 784-795. doi: 10.1136/jnnp-2012-303144

Zhu, X., Castellani, R., Takeda, A., Nunomura, A., Atwood, C., Perry, G., et al. (2001). Differential activation of neuronal ERK, JNK/SAPK and p38 in Alzheimer disease: the "two hit" hypothesis. Mech. Ageing Dev. 123, 39-46. doi: 10.1016/s0047-6374(01)00342-6

Zucker, S., Hymowitz, M., Conner, C., Zarrabi, H. M., Hurewitz, A. N., Matrisian, L., et al. (1999). Measurement of matrix metalloproteinases and tissue inhibitors of metalloproteinases in blood and tissues. clinical and experimental applications. Ann. N.Y. Acad. Sci. 878, 212-227. doi: 10.1111/j.1749-6632.1999. tb07687.x

Conflict of Interest Statement: EJM consults for RegenxBio. MDI discloses consultant fees at GE Healthcare. FML and SMM are listed as inventors on patents relating to LM11A-31, which are assigned to the University of North Carolina, University of California (UC), San Francisco and the Department of Veterans Affairs (VA). FML and SMM are entitled to royalties distributed by UC and the VA per their standard agreements. FML is a principal of, and has a financial interest in PharmatrophiX, a company focused on the development of small molecule ligands for neurotrophin receptors which has licensed several of these patents. The funders had no role in study design, data collection and analysis, decision to publish, or preparation of the manuscript.

The remaining authors declare that the research was conducted in the absence of any commercial or financial relationships that could be construed as a potential conflict of interest.

Copyright (c) 2019 Mufson, Counts, Ginsberg, Mahady, Perez, Massa, Longo and Ikonomovic. This is an open-access article distributed under the terms of the Creative Commons Attribution License (CC BY). The use, distribution or reproduction in other forums is permitted, provided the original author(s) and the copyright owner(s) are credited and that the original publication in this journal is cited, in accordance with accepted academic practice. No use, distribution or reproduction is permitted which does not comply with these terms. 